\title{
Core fragmentation and Toomre stability analysis of $\mathrm{W} 3\left(\mathrm{H}_{2} \mathrm{O}\right)$ A case study of the IRAM NOEMA large program CORE $\star, \star \star$
}

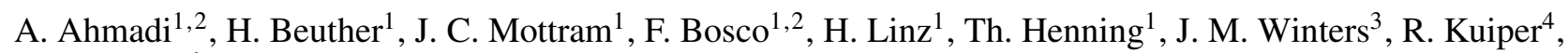 \\ R. Pudritz ${ }^{5}$, Á. Sánchez-Monge ${ }^{6}$, E. Keto ${ }^{7}$, M. Beltran ${ }^{8}$, S. Bontemps ${ }^{9}$, R. Cesaroni ${ }^{8}$, T. Csengeri ${ }^{10}$, S. Feng ${ }^{11}$, \\ R. Galvan-Madrid ${ }^{12}$, K. G. Johnston ${ }^{13}$, P. Klaassen ${ }^{14}$, S. Leurini ${ }^{15}$, S. N. Longmore ${ }^{16}$, S. Lumsden ${ }^{13}$, L. T. Maud ${ }^{17}$, \\ K. M. Menten ${ }^{10}$, L. Moscadelli ${ }^{8}$, F. Motte ${ }^{18}$, A. Palau ${ }^{12}$, T. Peters ${ }^{19}$, S. E. Ragan ${ }^{20}$, P. Schilke ${ }^{6}$, J. S. Urquhart ${ }^{21}$, \\ F. Wyrowski ${ }^{10}$, and H. Zinnecker ${ }^{22,23}$
}

(Affiliations can be found after the references)

Received 24 December 2017 / Accepted 21 July 2018

\begin{abstract}
Context. The fragmentation mode of high-mass molecular clumps and the properties of the central rotating structures surrounding the most luminous objects have yet to be comprehensively characterised.

Aims. We study the fragmentation and kinematics of the high-mass star-forming region $\mathrm{W} 3\left(\mathrm{H}_{2} \mathrm{O}\right)$, as part of the IRAM NOrthern Extended Millimeter Array (NOEMA) large programme CORE.

Methods. Using the IRAM NOEMA and the IRAM $30 \mathrm{~m}$ telescope, the CORE survey has obtained high-resolution observations of 20 well-known highly luminous star-forming regions in the $1.37 \mathrm{~mm}$ wavelength regime in both line and dust continuum emission. Results. We present the spectral line setup of the CORE survey and a case study for W3 $\left(\mathrm{H}_{2} \mathrm{O}\right)$. At $\sim 0$ '.35 (700 AU at $\left.2.0 \mathrm{kpc}\right)$ resolution, the $\mathrm{W} 3\left(\mathrm{H}_{2} \mathrm{O}\right)$ clump fragments into two cores (west and east), separated by $\sim 2300 \mathrm{AU}$. Velocity shifts of a few km s $\mathrm{s}^{-1}$ are observed in the dense-gas tracer, $\mathrm{CH}_{3} \mathrm{CN}$, across both cores, consistent with rotation and perpendicular to the directions of two bipolar outflows, one emanating from each core. The kinematics of the rotating structure about $\mathrm{W} 3\left(\mathrm{H}_{2} \mathrm{O}\right) \mathrm{W}$ shows signs of differential rotation of material, possibly in a disk-like object. The observed rotational signature around $\mathrm{W} 3\left(\mathrm{H}_{2} \mathrm{O}\right) \mathrm{E}$ may be due to a disk-like object, an unresolved binary (or multiple) system, or a combination of both. We fit the emission of $\mathrm{CH}_{3} \mathrm{CN}\left(12_{K}-11_{K}\right), K=4-6$ and derive a gas temperature map with a median temperature of $\sim 165 \mathrm{~K}$ across $\mathrm{W} 3\left(\mathrm{H}_{2} \mathrm{O}\right)$. We create a Toomre $Q$ map to study the stability of the rotating structures against gravitational instability. The rotating structures appear to be Toomre unstable close to their outer boundaries, with a possibility of further fragmentation in the differentially rotating core, $\mathrm{W} 3\left(\mathrm{H}_{2} \mathrm{O}\right) \mathrm{W}$. Rapid cooling in the Toomre unstable regions supports the fragmentation scenario.

Conclusions. Combining millimetre dust continuum and spectral line data toward the famous high-mass star-forming region $\mathrm{W} 3\left(\mathrm{H}_{2} \mathrm{O}\right)$, we identify core fragmentation on large scales, and indications for possible disk fragmentation on smaller spatial scales.
\end{abstract}

Key words. stars: formation - stars: massive - stars: early-type - stars: kinematics and dynamics stars: individual: $\mathrm{W} 3(\mathrm{H} 2 \mathrm{O}) /(\mathrm{OH})-$ techniques: interferometric

\section{Introduction}

Fundamental questions pertaining to the fragmentation of high-mass clumps and the accretion processes that result in the birth of the most massive stars $\left(M \gtrsim 8 M_{\odot}\right)$ still remain unanswered. This is in part due to the clustered nature of star formation and the typically large distances involved. For a long time the existence of high-mass stars had been puzzling as it was thought that the expected intense radiation pressure would prevent the accretion of enough material onto the protostar (e.g. Kahn 1974; Wolfire \& Cassinelli 1987). More recently, twoand three-dimensional (magneto)hydrodynamical simulations of collapsing cores have validated the need for accretion disks in the formation of very massive stars, analogous to low-mass star

\footnotetext{
${ }^{\star}$ Based on observations from an IRAM large program. IRAM is supported by INSU/CNRS (France), MPG (Germany), and IGN (Spain).

${ }^{\star \star}$ Observational data are only available at the CDS via anonymous ftp to cdsarc.u-strasbg.fr $(130.79 .128 .5)$ or via http://cdsarc.u-strasbg.fr/viz-bin/qcat?J/A+A/618/A46, or alternatively at http://www.mpia.de/core.
}

formation (e.g. Yorke \& Sonnhalter 2002; Krumholz et al. 2009; Kuiper et al. 2010, 2011; Peters et al. 2010; Kuiper \& Yorke 2013; Klassen et al. 2016). Furthermore, different fragmentation processes can contribute to the final stellar mass distribution within a single region, including fragmentation from clouds down to core scales (e.g. Bontemps et al. 2010; Palau et al. 2013, 2015; Beuther et al. 2018; see review by Motte et al. 2018) and disk fragmentation at smaller spatial scales (e.g. Matsumoto \& Hanawa 2003; see review by Kratter \& Lodato 2016).

In the disk-mediated accretion scenario, the non-isotropic treatment of the radiation field reduces the effect of radiation pressure in the radial direction, such that radiation can escape through the poles along the disk rotation axis, while the disk is shielded due to the high densities. Observationally, the existence of these disks is expected due to ubiquitous observations of collimated outflows (e.g. Beuther et al. 2002; Fallscheer et al. 2009; Leurini et al. 2011; Frank et al. 2014; Maud et al. 2015), which have also been predicted by theoretical models (e.g. Pudritz et al. 2007). Although some accretion disks in differential, Keplerian-like rotation about B-type (proto)stars have 
been found in recent years (e.g. Carrasco-González et al. 2012; Sánchez-Monge et al. 2013; Beltrán et al. 2014; see reviews by Cesaroni et al. 2007 and Beltrán \& de Wit 2016), the existence of such rotating structures around the most massive, O-type protostars is still elusive; only a few cases have been reported to date (Johnston et al. 2015; Ilee et al. 2016; Cesaroni et al. 2017).

As higher resolution observations are becoming more accessible, thus allowing structures to be resolved on scales $<1000 \mathrm{AU}$, it is important to determine whether disks around intermediate to high-mass stars (OB-type) are ubiquitous, and if so, to characterise their properties. What is the typical extent of these disks? Are they in differential rotation about a centrally dominating protostar, similar to their low-mass counterparts, and if so, over what range of radii? Is there any scale where a core stops fragmenting ${ }^{1}$ ? At what scales do we see the fragmentation of disks? Are close binary/multiple systems an outcome of disk fragmentation (as suggested by e.g. Meyer et al. 2018)? If so, stability analyses of these high-mass rotating cores and disks are needed to shed light on fragmentation at disk scales. These questions can only be answered with a statistical approach for a large sample of high-mass star-forming regions.

We have undertaken a large programme at IRAM, called CORE (Beuther et al. 2018), making use of the IRAM NOrthern Extended Millimeter Array (NOEMA, formerly Plateau de Bure Interferometer) at $1.37 \mathrm{~mm}$ in both line and continuum emission to study the early phases of star formation for a sample of 20 highly luminous $\left(L>10^{4} L_{\odot}\right)$ star-forming regions at high angular resolution $\left(\sim 0.4^{\prime \prime}\right)$ to analyse their fragmentation and characterise the properties of possible rotating structures. Additionally, observations with the IRAM $30 \mathrm{~m}$ telescope are included to complement the interferometric data, allowing us to understand the role of the environment by studying high-mass star formation at scales larger than those covered by the interferometer. Observations in the $1.3 \mathrm{~mm}$ wavelength regime of the CORE project began in June 2014 and finished in January 2017 , consisting of a total of more than $400 \mathrm{~h}$ of observations with NOEMA. The sample selection criteria and initial results from the observed level of fragmentation in the full sample are presented in Beuther et al. (2018), and details of the $30 \mathrm{~m}$ observations and the merging of single-dish observations with interferometric observations can be found in Mottram et al. (2018). In this work, we describe our spectral setup and present a case study of one of the most promising star-forming clouds in our sample, $\mathrm{W} 3\left(\mathrm{H}_{2} \mathrm{O}\right)$.

$\mathrm{W} 3\left(\mathrm{H}_{2} \mathrm{O}\right)$, also known as the Turner-Welch object, resides in the W3 high-mass star-forming region and was initially identified through observations of the dense-gas tracer $\mathrm{HCN}$ at 88.6 GHz (Turner \& Welch 1984). It is located $\sim 0.05 \mathrm{pc}\left(5^{\prime \prime}\right)$ east of the well-known ultra compact H II region (UCHII) W3(OH). The name $\mathrm{W} 3\left(\mathrm{H}_{2} \mathrm{O}\right)$ stems from the existence of water masers in the vicinity of the source (Dreher \& Welch 1981), allowing for an accurate distance measurement of $2.0 \mathrm{kpc}$ for this region (Hachisuka et al. 2006; cf. Xu et al. 2006). The relative proper motions of these masers are further explained by an outflow model oriented in the east-west direction (Hachisuka et al. 2006). A continuum source elongated in the east-west direction and spanning the same extent as the water maser outflow has been observed in subarcsecond VLA observations in the radio regime with a spectral index of -0.6 , providing evidence for synchrotron emission (Reid et al. 1995; Wilner et al. 1999). This source of synchrotron emission has been characterised by

\footnotetext{
1 Here a core is defined as a gravitationally bound region that forms single or multiple stars, following Williams et al. (2000).
}

a jet-like model due to its morphology, and the point symmetry of its wiggly bent structure about the centre hints at the possibility of jet precession. Moreover, Shchekinov \& Sobolev (2004) attribute this radio emission to a circumstellar jet or wind ionised by the embedded (proto)star at this position. Additional radio continuum sources have been detected in the vicinity of the synchrotron jet, the closest of which is to the west of the elongated structure and has a spectral index of 0.9 (Wilner et al. 1999; Chen et al. 2006), consistent with a circumstellar wind being ionised by another embedded protostellar source. In fact, the high angular resolution ( 0.'7) observations of Wyrowski et al. (1999) in the $1.36 \mathrm{~mm}$ band allowed for the detection of three continuum peaks in thermal dust emission, one of which peaks on the position of the water maser outflow and synchrotron jet, and another on the position of the radio continuum source with positive spectral index, confirming the existence of a second source at this position. The detection of two bipolar molecular (CO) outflows further supports the protobinary scenario, suggesting that $\mathrm{W} 3\left(\mathrm{H}_{2} \mathrm{O}\right)$ may be harbouring at least two rotating structures (Zapata et al. 2011). The two cores within $\mathrm{W} 3\left(\mathrm{H}_{2} \mathrm{O}\right)$ have individual luminosities on the order of $2 \times 10^{4} L_{\odot}$, suggesting two $15 M_{\odot}$ stars of spectral type B0 (see Sect. 4.3).

In this paper, we study the fragmentation properties of $\mathrm{W} 3\left(\mathrm{H}_{2} \mathrm{O}\right)$ and the kinematics of the rotating structures within it. We use this source as a test-bed for an expanded study in a forthcoming paper which will focus on the kinematic properties of a larger sample within our survey. The structure of the paper is as follows. Section 2 presents our spectral line setup within the CORE survey with the details of our observations and data reduction for $\mathrm{W} 3\left(\mathrm{H}_{2} \mathrm{O}\right)$. The observational results are described in Sect. 3. The kinematics, temperature, and stability analysis of $\mathrm{W} 3\left(\mathrm{H}_{2} \mathrm{O}\right)$ is presented in Sect. 4. The main findings are summarised in Sect. 5.

\section{Observations and data reduction}

\subsection{NOEMA observations}

Observations of $\mathrm{W} 3\left(\mathrm{H}_{2} \mathrm{O}\right)$ at $1.37 \mathrm{~mm}$ were made between October 2014 and March 2016 in the A-, B-, and D-array configurations of NOEMA in track-sharing mode with W3 IRS4. The compact D-array observations were made with six antennas, while seven antennas were used for the more extended Aand B-array observations. Baselines in the range of 19-760 m were covered, therefore, the NOEMA observations are not sensitive to structures larger than $12^{\prime \prime}(0.1 \mathrm{pc})$ at $220 \mathrm{GHz}$. On-source observations were taken in roughly 20 min increments distributed over an observing run and interleaved with observations of various calibration sources. The phase centre for the observations of $\mathrm{W} 3\left(\mathrm{H}_{2} \mathrm{O}\right)$ is $\alpha(\mathrm{J} 2000)=02^{\mathrm{h}} 27^{\mathrm{m}} 03^{\mathrm{s}} .87, \delta(\mathrm{J} 2000)=$ $61^{\circ} 52^{\prime} 24^{\prime \prime} 5$. A summary of the observations can be found in Table 1.

The full CORE sample of 20 regions has been observed simultaneously with a narrow- and a wide-band correlator. The wide-band correlator, WideX, has four units, each with $1.8 \mathrm{GHz}$ bandwidth, covering two overlapping ranges in frequency in both horizontal and vertical polarisations ( $\mathrm{H}$ and $\mathrm{V}$ ) with a fixed spectral resolution of $1.95 \mathrm{MHz}\left(\sim 2.7 \mathrm{~km} \mathrm{~s}^{-1}\right.$ at $\left.219 \mathrm{GHz}\right)$. The full coverage of the WideX correlator is shown in Fig. 1 with bright lines marked. The narrow-band correlator has eight units, each with $80 \mathrm{MHz}$ bandwidth and a spectral resolution of $0.312 \mathrm{MHz}$ $\left(\sim 0.43 \mathrm{~km} \mathrm{~s}^{-1}\right)$, placed in the $1.37 \mathrm{~mm}$ wavelength regime. The frequency coverage of the correlator bands are listed in Table 2. The narrow-band correlator can only process the signal from six 


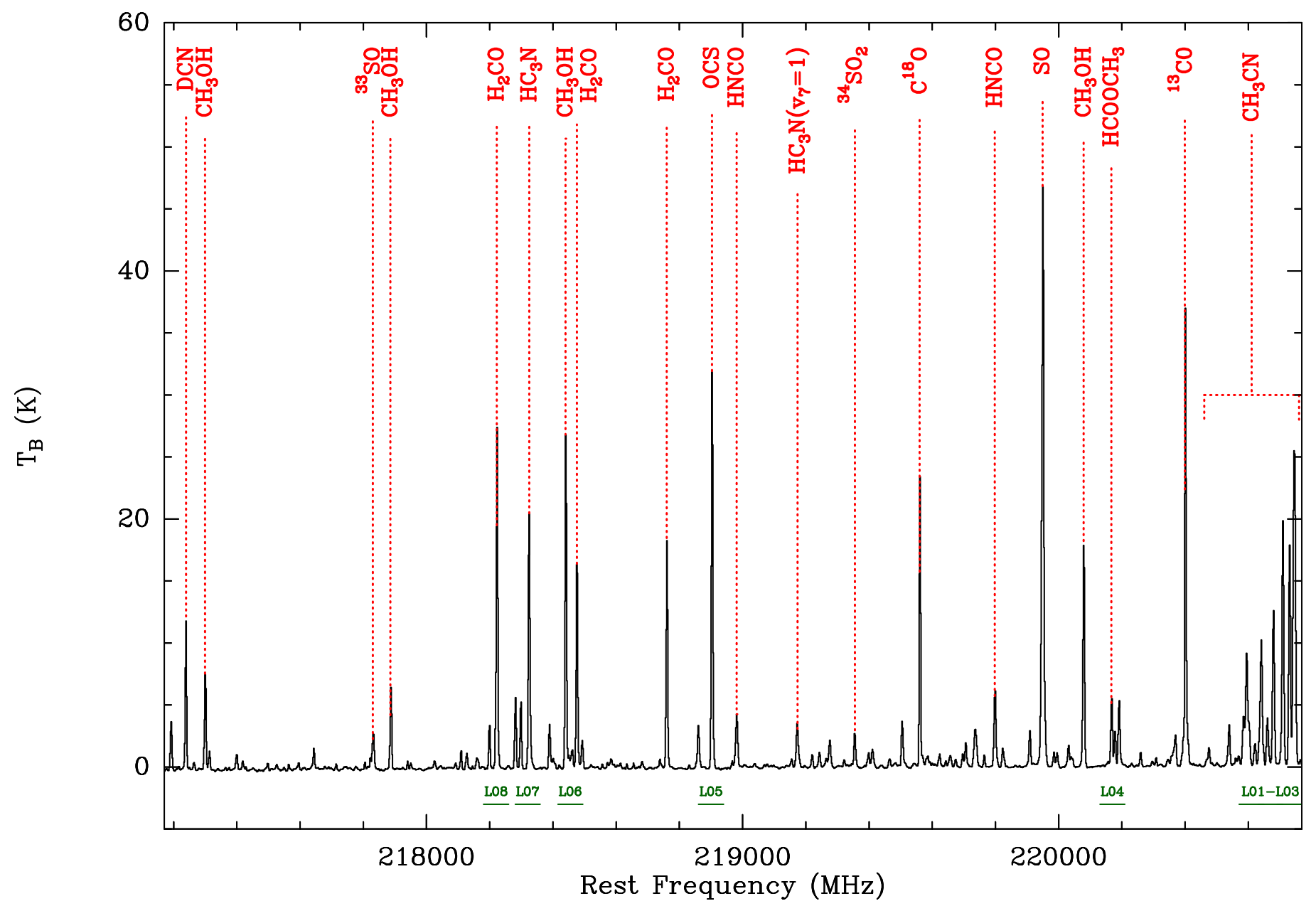

Fig. 1. Full WideX spectrum of $\mathrm{W} 3\left(\mathrm{H}_{2} \mathrm{O}\right)$ averaged over a $4^{\prime \prime} \times 4^{\prime \prime}$ region encompassing two cores, W3 $\left(\mathrm{H}_{2} \mathrm{O}\right) \mathrm{E}$ and W3( $\left.\mathrm{H}_{2} \mathrm{O}\right) \mathrm{W}$, showing the chemical richness of the source. The coverage of the narrow-band correlator units are shown as horizontal green lines and labelled accordingly. The units of the spectrum have been converted from Jy beam ${ }^{-1}$ to $\mathrm{K}$ by multiplying the flux by $188 \mathrm{~K} \mathrm{Jy}^{-1}$ under the Rayleigh-Jeans approximation.

Table 1. Observations of $\mathrm{W} 3\left(\mathrm{H}_{2} \mathrm{O}\right)$ and $\mathrm{W} 3(\mathrm{OH})$.

\begin{tabular}{lccc}
\hline Observation date & Array & $\begin{array}{c}\text { Time on-source } \\
\text { (h) }\end{array}$ & $\begin{array}{c}\text { Bandpass } \\
\text { calibrator }\end{array}$ \\
\hline 2014-October-31 & D & 3.9 & $3 \mathrm{C} 454.3$ \\
2015-March-18 & A & 2.6 & $3 \mathrm{C} 84$ \\
2015-April-3 & B & 0.9 & $3 \mathrm{C} 84$ \\
2015-April-6 & B & 1.3 & $3 \mathrm{C} 84$ \\
2016-March-11 & A & 2.2 & $3 \mathrm{C} 84$ \\
\hline
\end{tabular}

Notes. The phase and flux calibrators were 0059+581 and MWC349, respectively, for all observations.

antennas; therefore, in cases where the sources were observed with more than six antennas, the correlator automatically accepts the signal from the antennas that yield the best $u v$-coverage. Important lines covered by the narrow-band receiver are listed in Table 3 and presented in Fig. 2 for the pixel at the phase centre toward $\mathrm{W} 3\left(\mathrm{H}_{2} \mathrm{O}\right)$.

Data reduction and imaging were performed with the CLIC and MAPPING program of the GILDAS $^{2}$ software package developed by the IRAM and Observatoire de Grenoble. The

\footnotetext{
2 http://wWW.iram.fr/IRAMFR/GILDAS
}

Table 2. Correlator units and frequency ranges observed with NOEMA.

\begin{tabular}{lccc}
\hline \hline Correlator & Spectral unit & Pol. & $\begin{array}{c}\text { Frequency range } \\
(\mathrm{MHz})\end{array}$ \\
\hline Narrow-band & L01 & H & $220690.6-220769.7$ \\
& L02 & H & $220630.6-220709.7$ \\
& L03 & H & $220570.6-220649.7$ \\
& L04 & H & $220130.6-220209.7$ \\
& L05 & H & $218860.6-218939.7$ \\
& L06 & H & $218415.6-218494.7$ \\
L07 & H & $218280.6-218359.7$ \\
LideX & L08 & H & $218180.6-218259.7$ \\
\hline L10 & H & 218 878.6-220 859.5 \\
& L11 & H & $217078.6-219059.4$ \\
\hline
\end{tabular}

Notes. $\mathrm{H}$ and $\mathrm{V}$ correspond to horizontal and vertical polarisations.

continuum was extracted by identifying line-free channels in the range $217078.6-220859.5 \mathrm{MHz}$ covered by all four spectral units of the WideX correlator. As we are interested in achieving the highest possible angular resolution, we CLEANed 

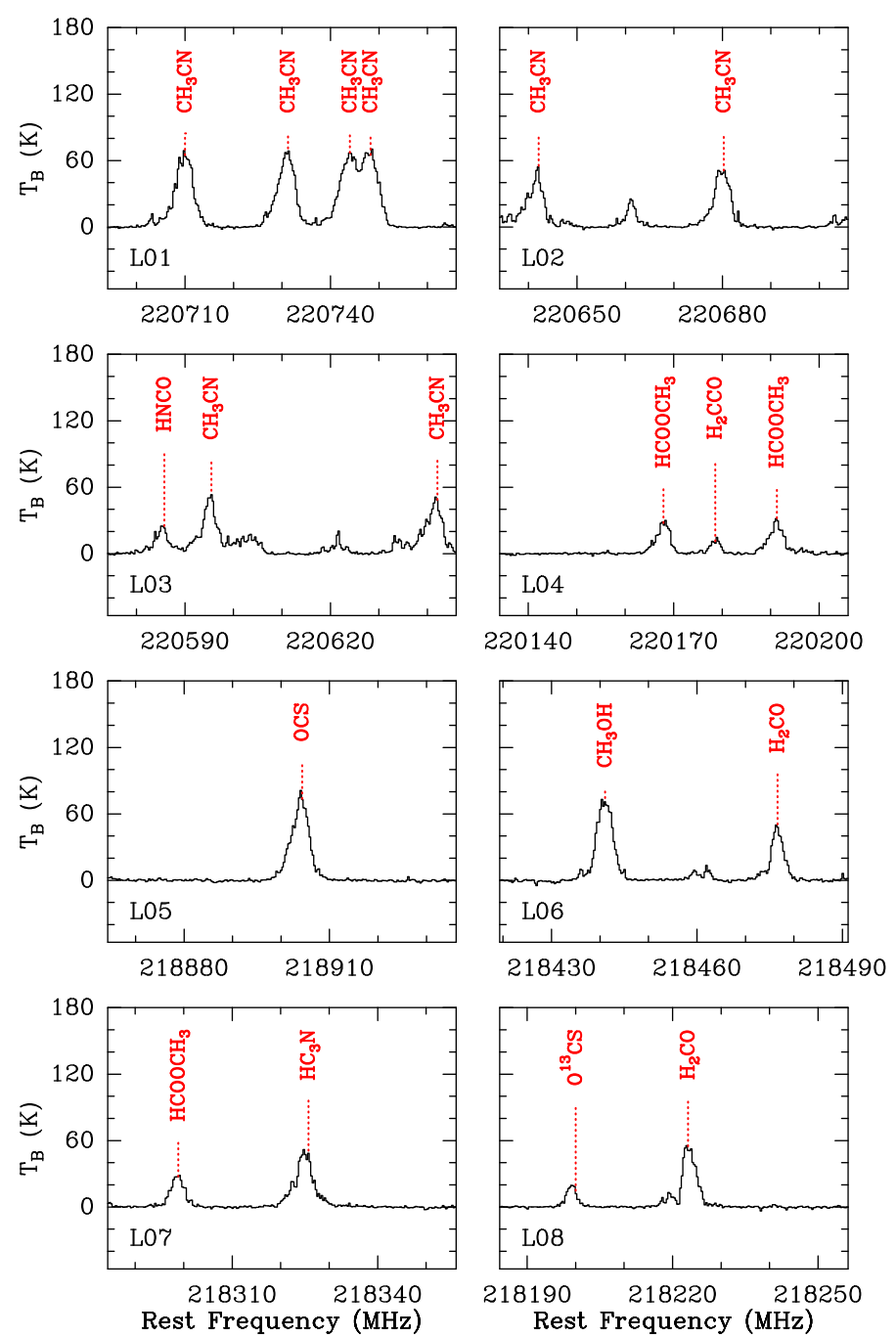

Fig. 2. Spectra of the frequency range covered by the narrow-band correlator for the pixel at the phase centre toward $\mathrm{W} 3\left(\mathrm{H}_{2} \mathrm{O}\right)$. The name of the correlator unit is listed in the bottom left corner of each panel; some of the detected lines are marked.

the cubes using the CLARK algorithm (Clark 1980) with a uniform weighting (robust parameter of 0.1$)^{3}$ yielding a synthesised beam size of $0^{\prime} .43 \times 0^{\prime} .^{\prime} 32, \mathrm{PA}=86^{\circ}$, and an rms noise of $3.2 \mathrm{mJy} \mathrm{beam}^{-1}$ for the continuum emission using the combined set of observations in the A-, B-, and D-array configurations (hereafter $\mathrm{ABD}$ ). We also imaged the data from just the A- and $\mathrm{B}$-array configurations (hereafter $\mathrm{AB}$ ), as well as the A-array only, for which the synthesised beam sizes and rms noise values are summarised in Table 4.

Continuum subtraction for the lines was performed in the $u v$-plane by subtracting the emission in the line-free channels in the spectral unit in which the line was observed. Due to line contamination in spectral unit L03, we used the continuum from spectral unit L02 to remove the continuum from spectral unit L03, under the assumption that there is no significant spectral slope between the two adjacent spectral windows. For the WideX continuum subtraction, we subtracted the continuum obtained from line-free channels in all four spectral units. All narrow-band spectra were resampled to a spectral resolution of $0.5 \mathrm{~km} \mathrm{~s}^{-1}$ and when imaged with the CLARK algorithm and uniform weighting

\footnotetext{
3 This corresponds to the CASA robust weighting with a robustness parameter of -2 .
}

Table 3. Bright lines covered in the narrow-band correlator setup.

\begin{tabular}{lccr}
\hline \hline Molecule & Transition & $\begin{array}{c}\text { Rest frequency } \\
(\mathrm{MHz})\end{array}$ & $\begin{array}{r}E_{u} / k \\
(\mathrm{~K})\end{array}$ \\
\hline $\mathrm{O}^{13} \mathrm{CS}$ & $18-17$ & 218199.00 & 99.5 \\
$\mathrm{H}_{2} \mathrm{CO}$ & $3_{0,3}-2_{0,2}$ & 218222.19 & 21.0 \\
$\mathrm{HCOOCH}$ & $17_{3,14}-16_{3,13} \mathrm{~A}$ & 218297.89 & 99.7 \\
$\mathrm{HC}_{3} \mathrm{~N}$ & $24-23$ & 218324.72 & 131.0 \\
$\mathrm{CH}_{3} \mathrm{OH}$ & $4-3$ & 218440.05 & 45.5 \\
$\mathrm{H}_{2} \mathrm{CO}$ & $3_{2,2}-2_{2,1}$ & 218475.63 & 68.1 \\
$\mathrm{OCS}$ & $18-17$ & 218903.36 & 99.81 \\
$\mathrm{HCOOCH}$ & $17_{4,13}-16_{4,12} \mathrm{E}$ & 220166.89 & 103.1 \\
$\mathrm{H}_{2} \mathrm{CCO}$ & $11_{1,11}-10_{1,10}$ & 220177.57 & 76.5 \\
$\mathrm{HCOOCH}$ & $17_{4,13}-16_{4,12} \mathrm{~A}$ & 220190.29 & 103.2 \\
$\mathrm{HNCO}$ & $10_{1,9}-9_{1,8}$ & 220584.75 & 101.5 \\
$\mathrm{CH}_{3} \mathrm{CN}$ & $12_{6}-11_{6}$ & 220594.42 & 325.9 \\
$\mathrm{CH}_{3}^{13} \mathrm{CN}$ & $12_{3}-11_{3}$ & 220599.98 & 133.1 \\
$\mathrm{CH}_{3}^{13} \mathrm{CN}$ & $12_{2}-11_{2}$ & 220621.14 & 97.4 \\
$\mathrm{CH}_{3}^{13} \mathrm{CN}$ & $12_{1}-11_{1}$ & 220633.83 & 76.0 \\
$\mathrm{CH}_{3}^{13} \mathrm{CN}$ & $12_{0}-11_{0}$ & 220638.07 & 68.8 \\
$\mathrm{CH}_{3} \mathrm{CN}$ & $12_{5}-11_{5}$ & 220641.08 & 247.4 \\
$\mathrm{CH}_{3} \mathrm{CN}$ & $12_{4}-11_{4}$ & 220679.29 & 183.2 \\
$\mathrm{CH}_{3} \mathrm{CN}$ & $12_{3}-11_{3}$ & 220709.02 & 133.2 \\
$\mathrm{CH}_{3} \mathrm{CN}$ & $12_{2}-11_{2}$ & 220730.26 & 97.4 \\
$\mathrm{CH}_{3} \mathrm{CN}$ & $12_{1}-11_{1}$ & 220743.01 & 76.0 \\
$\mathrm{CH}_{3} \mathrm{CN}$ & $12_{0}-11_{0}$ & 220747.26 & 68.9 \\
\hline
\end{tabular}

Notes. Rest frequencies and upper energy levels have been obtained from the Cologne Database for Molecular Spectroscopy (CDMS; Müller et al. 2001, 2005) with the exception of those for $\mathrm{HCOOCH}_{3}$ transitions, which were acquired from the Jet Propulsion Laboratory (Pickett et al. 1998).

have a negligibly smaller synthesised beam than the continuum images. The average rms noise of the line images in the ABD configuration is $11.2 \mathrm{mJy}$ beam ${ }^{-1} \mathrm{~km} \mathrm{~s}^{-1}$. The synthesised beam size and the average rms noise of the line data for all imaged combinations of array configurations are listed in Table 4.

\subsection{IRAM 30 m observations}

Observations of $\mathrm{W} 3\left(\mathrm{H}_{2} \mathrm{O}\right)$ with the $30 \mathrm{~m}$ telescope were obtained on March 13, 2015, centred on the same position as the phase centre of the interferometric observations. We used the Eight MIxer Receiver (EMIR) covering the range 213-236 GHz, reaching a spectral resolution of $0.3 \mathrm{~km} \mathrm{~s}^{-1}$. In this work, we have merged the NOEMA observations of ${ }^{13} \mathrm{CO}$ with the single-dish observations using the MAPPING software and CLEANed the merged cube with the Steer-Dewdney-Ito (SDI) method (Steer et al. 1984) in order to recover more of the extended features to study molecular outflows. Further details of the $30 \mathrm{~m}$ observations and data reduction, as well as the merging process, can be found in Mottram et al. (2018). The resulting merged image has an angular resolution of $1^{\prime \prime} .14 \times 0 .{ }^{\prime} 92, \mathrm{PA}=49^{\circ}$, and an rms noise of $8.4 \mathrm{mJy}_{\text {beam }}{ }^{-1} \mathrm{~km} \mathrm{~s}^{-1}$. We also make use of our singledish ${ }^{13} \mathrm{CO}$ data which have been reduced and converted to brightness temperatures for a detailed outflow analysis (see Sect. 3.3).

\section{Observational results}

In the following, we present our detailed analysis for $\mathrm{W} 3\left(\mathrm{H}_{2} \mathrm{O}\right)$, and when applicable, we also showcase our observational results for $\mathrm{W} 3(\mathrm{OH})$. Our analysis mainly uses the continuum and 
Table 4. Details of CLEANed images.

\begin{tabular}{|c|c|c|c|c|}
\hline \multirow[b]{2}{*}{ Configuration } & \multicolumn{2}{|c|}{ Continuum } & \multicolumn{2}{|c|}{ Line (Narrow-band) } \\
\hline & $\begin{array}{l}\text { Synthesised } \\
\text { beam }\end{array}$ & $\begin{array}{c}\text { rms noise } \\
\left(\mathrm{mJy}^{-} \text {beam }^{-1}\right)\end{array}$ & $\begin{array}{l}\text { Synthesised } \\
\text { beam }\end{array}$ & $\begin{array}{c}\text { Average rms noise } \\
\left(\mathrm{mJy} \mathrm{beam}^{-1} \mathrm{~km} \mathrm{~s}^{-1}\right)\end{array}$ \\
\hline $\mathrm{ABD}$ & $0^{\prime} 43 \times 00^{\prime} 32, \mathrm{PA}=86^{\circ}$ & 3.2 & $0 .^{\prime} 42 \times 0 . .^{\prime} 31, \mathrm{PA}=87^{\circ}$ & 11.2 \\
\hline $\mathrm{AB}$ & $0.41 \times 00^{\prime} 30, \mathrm{PA}=86^{\circ}$ & 2.6 & $0.38 \times 0 . ' 28, \mathrm{PA}=87^{\circ}$ & 8.6 \\
\hline $\mathrm{A}$ & $0.39 \times 0 . \prime 28, \mathrm{PA}=88^{\circ}$ & 2.5 & $0.36 \times 0 . ' 26, \mathrm{PA}=88^{\circ}$ & 8.0 \\
\hline
\end{tabular}

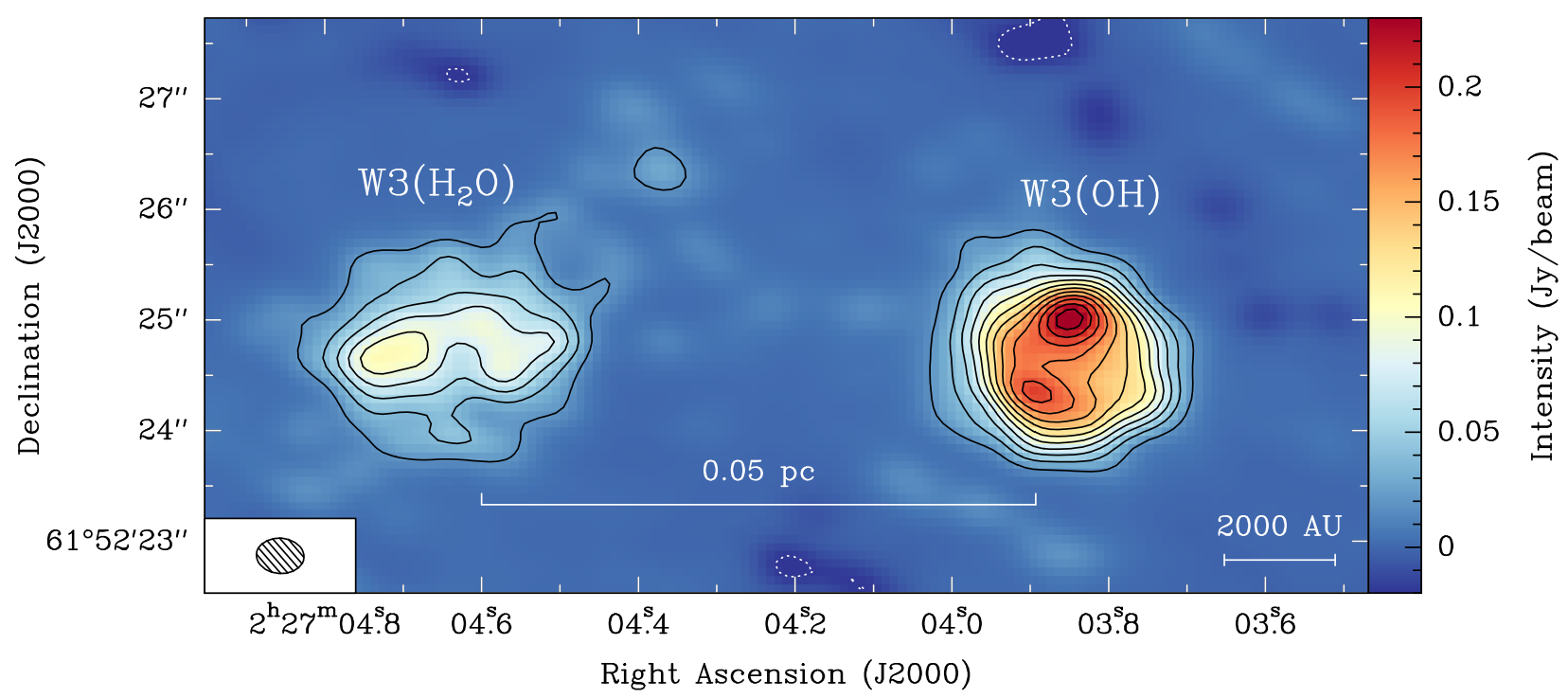

Fig. 3. NOEMA $1.37 \mathrm{~mm}(219 \mathrm{GHz})$ continuum image toward $\mathrm{W} 3\left(\mathrm{H}_{2} \mathrm{O}\right)$ and $\mathrm{W} 3(\mathrm{OH})$ in the ABD configuration. The solid contours start at $6 \sigma$ and increase in steps of $6 \sigma\left(1 \sigma=3.2 \mathrm{mJy} \mathrm{beam}^{-1}\right)$. The dotted contours show the same negative levels. A scale bar and the synthesised beam $\left(0 ., 43 \times 0 . \prime 32, \mathrm{PA}=86^{\circ}\right)$ are shown at the bottom.

$\mathrm{CH}_{3} \mathrm{CN}$ spectral line emission. Maps of the other lines are shown in Appendix A.

\subsection{Continuum emission}

Figure 3 shows the $1.37 \mathrm{~mm}(219 \mathrm{GHz})$ continuum emission map of $\mathrm{W} 3\left(\mathrm{H}_{2} \mathrm{O}\right)$ and $\mathrm{W} 3(\mathrm{OH})$ in the ABD configuration. At this wavelength, the continuum emission in our field of view is dominated in $\mathrm{W} 3(\mathrm{OH})$ by free-free emission, while the emission in $\mathrm{W} 3\left(\mathrm{H}_{2} \mathrm{O}\right)$ is due to dust (Wyrowski et al. 1999). In the following, we focus on the fragmentation and kinematics of the younger region, $\mathrm{W} 3\left(\mathrm{H}_{2} \mathrm{O}\right)$.

Figure 4 shows a comparison of the continuum emission maps of $\mathrm{W} 3\left(\mathrm{H}_{2} \mathrm{O}\right)$ obtained by imaging the $\mathrm{ABD}, \mathrm{AB}$, and A-array only observations. The integrated flux within the $6 \sigma$ contours is 1220,656 , and $364 \mathrm{mJy}$ for the $\mathrm{ABD}, \mathrm{AB}$, and A-array images, respectively. The fragmentation of $\mathrm{W} 3\left(\mathrm{H}_{2} \mathrm{O}\right)$ into two cores, separated by $2300 \mathrm{AU}$, is best seen in the $\mathrm{AB}$ image at 700 AU scales. The two cores are labelled $\mathrm{W} 3\left(\mathrm{H}_{2} \mathrm{O}\right)$ $\mathrm{E}$ and $\mathrm{W} 3\left(\mathrm{H}_{2} \mathrm{O}\right) \mathrm{W}$, and their peak continuum positions are depicted by stars in Fig. 4, marking the positions of embedded (proto)stars. The peak position of $\mathrm{W} 3\left(\mathrm{H}_{2} \mathrm{O}\right) \mathrm{E}$ is $\alpha(\mathrm{J} 2000)=$ $02^{\mathrm{h}} 27^{\mathrm{m}} 04^{\mathrm{s}} .73, \delta(\mathrm{J} 2000)=61^{\circ} 52^{\prime} 24^{\prime \prime} .66$, and that of $\mathrm{W} 3\left(\mathrm{H}_{2} \mathrm{O}\right) \mathrm{W}$ is $\alpha(\mathrm{J} 2000)=02^{\mathrm{h}} 27^{\mathrm{m}} 04^{\mathrm{s}} .57, \quad \delta(\mathrm{J} 2000)=61^{\circ} 52^{\prime} 24^{\prime \prime} .59$. The approximate separation boundary between $\mathrm{W} 3\left(\mathrm{H}_{2} \mathrm{O}\right)$ E and $\mathrm{W}$ is marked with a vertical dashed line. The integrated flux within $6 \sigma$ contours and the separation boundary are 377 and $279 \mathrm{mJy}$ for $\mathrm{W} 3\left(\mathrm{H}_{2} \mathrm{O}\right) \mathrm{E}$ and $\mathrm{W} 3\left(\mathrm{H}_{2} \mathrm{O}\right) \mathrm{W}$, respectively. Furthermore, there is an additional emission peak to the northwest of $\mathrm{W} 3\left(\mathrm{H}_{2} \mathrm{O}\right)$ at an offset of $-1 .{ }^{\prime \prime} 6,11^{\prime \prime} 6(\alpha(\mathrm{J} 2000)=$ $\left.02^{\mathrm{h}} 27^{\mathrm{m}} 04^{\mathrm{s}} .37, \delta(\mathrm{J} 2000)=61^{\circ} 52^{\prime} 26^{\prime \prime} 35\right)$, which is best seen in the ABD image as it has the best sensitivity. This is most likely a site for the formation of lower mass stars.

Radiative transfer models by Chen et al. (2006) for $\mathrm{W} 3\left(\mathrm{H}_{2} \mathrm{O}\right)$ in the $1.4 \mathrm{~mm}$ wavelength regime show that the averaged dust optical depth is less than 0.09; therefore, we assume the thermal dust emission to be optically thin in our observations. The positions of continuum peaks $\mathrm{A}$ and $\mathrm{C}$ from Wyrowski et al. (1999) coincide well with the continuum peaks $\mathrm{W} 3\left(\mathrm{H}_{2} \mathrm{O}\right) \mathrm{E}$ and $\mathrm{W} 3\left(\mathrm{H}_{2} \mathrm{O}\right) \mathrm{W}$ in our observations to within a synthesised beam (see Fig. 4). Wyrowski et al. (1999) had attributed the third peak in their observations between the other two cores to an interplay of high column density and low temperatures in the central region, and concluded that $\mathrm{W} 3\left(\mathrm{H}_{2} \mathrm{O}\right)$ is harbouring two cores at the positions of continuum peaks $\mathrm{A}$ and $\mathrm{C}$. Our double-peaked continuum image in $\mathrm{AB}$, with a better spatial resolution than that of Wyrowski et al. (1999) by a factor of 3.3, supports this interpretation. Furthermore, peak B coincides well with the northeast extension of $\mathrm{W} 3\left(\mathrm{H}_{2} \mathrm{O}\right) \mathrm{W}$ in our observations, best seen in the middle panel of Fig. 4.

\subsection{Line emission}

$\mathrm{W} 3\left(\mathrm{H}_{2} \mathrm{O}\right)$ is one of the most chemically rich sources in our sample (see Fig. 1) with detections of sulphur-bearing species such as ${ }^{33} \mathrm{SO}$ and ${ }^{34} \mathrm{SO}_{2}$, complex species such as $\mathrm{HCOOCH}_{3}$, and vibrationally excited lines of $\mathrm{HC}_{3} \mathrm{~N}$, among many others. Figure 5 shows integrated intensity (zeroth moment) 


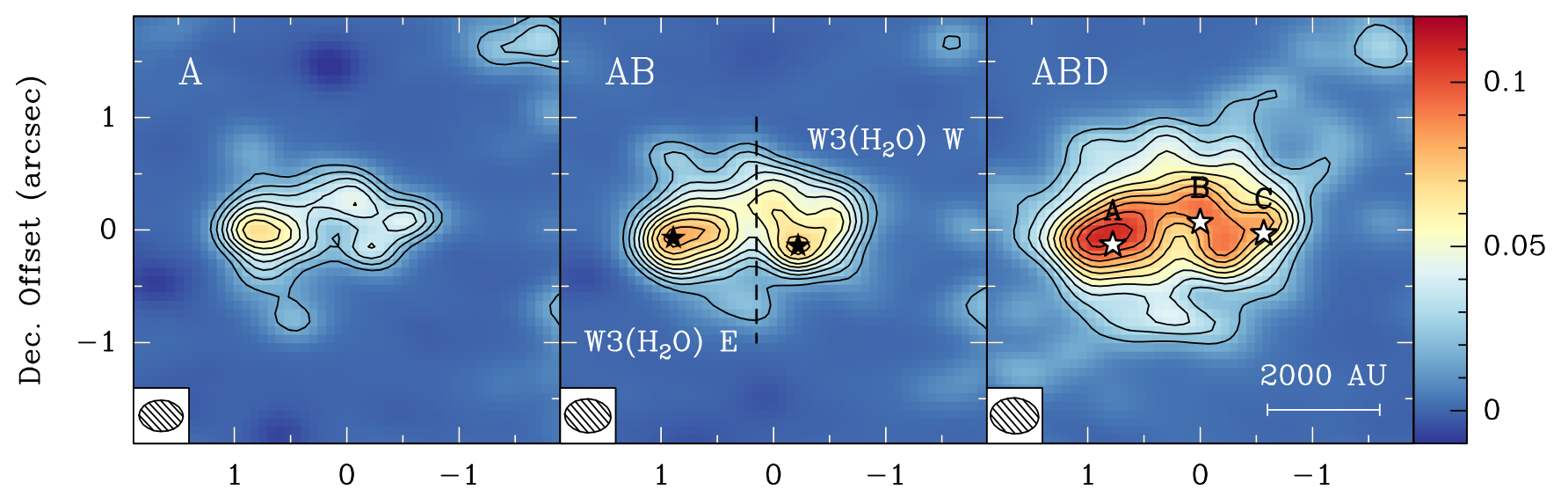

R.A. Offset (arcsec)

Fig. 4. 1.37 mm continuum image toward $\mathrm{W} 3\left(\mathrm{H}_{2} \mathrm{O}\right)$ observed with the A (left panel), $\mathrm{AB}$ (middle panel), and ABD (right panel) configurations of NOEMA. The solid contours start at $6 \sigma$ and increase in steps of $3 \sigma$ (see Table 4). Synthesised beams are shown in the bottom left corners of each panel, along with a scale bar in the bottom right of the right-hand panel. The black stars in the middle panel correspond to the positions of the continuum peaks, marking the locations of the two individual cores, $\mathrm{W} 3\left(\mathrm{H}_{2} \mathrm{O}\right) \mathrm{W}$ and $\mathrm{W} 3\left(\mathrm{H}_{2} \mathrm{O}\right) \mathrm{E}$, with the dashed line as the approximate separation boundary. The white stars in the right panel correspond to the positions of the continuum peaks $\mathrm{A}$, B, and C from Wyrowski et al. (1999). The offset zero position is the phase centre of the observations: $\alpha(\mathrm{J} 2000)=02^{\mathrm{h}} 27^{\mathrm{m}} 03^{\mathrm{s}} \cdot 87, \delta(\mathrm{J} 2000)=61^{\circ} 52^{\prime} 24^{\prime \prime} .5$.

and intensity-weighted peak velocity (first moment) maps of $\mathrm{CH}_{3} \mathrm{CN}\left(12_{3}-11_{3}\right)$ for $\mathrm{W} 3\left(\mathrm{H}_{2} \mathrm{O}\right)$ and $\mathrm{W} 3(\mathrm{OH})$. The zeroth moment map confirms the fragmentation of $\mathrm{W} 3\left(\mathrm{H}_{2} \mathrm{O}\right)$ into two cores. The moment maps of most lines covered by the narrow-band receiver (see Table 3 and Fig. 2) are presented in Appendix A. All moment maps have been created inside regions where the signal-to-noise ratio $(\mathrm{S} / \mathrm{N})$ is greater than $5 \sigma$. The integrated intensity maps of most tracers for $\mathrm{W} 3\left(\mathrm{H}_{2} \mathrm{O}\right)$ also show two peaks coincident with the locations of $\mathrm{W} 3\left(\mathrm{H}_{2} \mathrm{O}\right) \mathrm{E}$ and $\mathrm{W} 3\left(\mathrm{H}_{2} \mathrm{O}\right) \mathrm{W}$. While the continuum emission is stronger for $\mathrm{W} 3\left(\mathrm{H}_{2} \mathrm{O}\right) \mathrm{E}$, some dense gas tracers (e.g. $\mathrm{CH}_{3} \mathrm{CN}, \mathrm{HC}_{3} \mathrm{~N}$ ) show stronger line emission towards $\mathrm{W} 3\left(\mathrm{H}_{2} \mathrm{O}\right) \mathrm{W}$.

The bottom panels in Fig. 5 show the intensity-weighted peak velocity (first moment) map of the region in $\mathrm{CH}_{3} \mathrm{CN}\left(12_{3}-11_{3}\right)$. We chose to do our kinematic analyses on this transition as it is the strongest unblended line in the methyl cyanide $\left(\mathrm{CH}_{3} \mathrm{CN}\right)$ $K$-ladder. There is a clear velocity gradient in the east-west direction across $\mathrm{W} 3\left(\mathrm{H}_{2} \mathrm{O}\right)$, and in the NW-SE direction across $\mathrm{W} 3(\mathrm{OH})$. The systemic velocities of both clumps are determined by averaging the spectra of $\mathrm{CH}_{3} \mathrm{CN}\left(12_{3}-11_{3}\right)$ over a $4^{\prime \prime} \times 4^{\prime \prime}$ area centred on each source and fitting a Gaussian line to the resulting averaged spectrum. In this way, $\mathrm{W} 3\left(\mathrm{H}_{2} \mathrm{O}\right)$ and $\mathrm{W} 3(\mathrm{OH})$ have average velocities of -49.1 and $-45.0 \mathrm{~km} \mathrm{~s}^{-1}$, respectively.

The velocity gradient across $\mathrm{W} 3\left(\mathrm{H}_{2} \mathrm{O}\right)$ is detected in most of the high spectral resolution lines in our survey (see Fig. A.2) and spans $\sim 6000 \mathrm{AU}$ in size, corresponding to an amplitude of $170 \mathrm{~km} \mathrm{~s}^{-1} \mathrm{pc}^{-1}$. The velocity gradient resolved in $\mathrm{W} 3(\mathrm{OH})$ has an amplitude of $\sim 100 \mathrm{~km} \mathrm{~s}^{-1} \mathrm{pc}^{-1}$ and is roughly perpendicular to the motion of the ionised gas in the east-west direction as traced by the H92 $\alpha$ line in observations of Keto et al. (1995), and is perpendicular to the direction of the "champagne flow" observed to the northeast at radio frequencies (Keto et al. 1995; Wilner et al. 1999). Thus, in W3(OH) we seem to be witnessing the large-scale motion of the remnant molecular gas. The interpretation of line emission for $\mathrm{W} 3(\mathrm{OH})$ is complex because most of the continuum emission at $1.37 \mathrm{~mm}$ is due to free-free emission which affects the appearance of molecular lines. In practice, free-free emission at $1.3 \mathrm{~mm}$ reduces the molecular line emission which is reflected by the reduced integrated line emission towards the peaks of $\mathrm{W} 3(\mathrm{OH})$. As this is beyond the scope of this paper, we refrain from further analysis of $\mathrm{W} 3(\mathrm{OH})$.

\subsection{Outflow structure}

In Fig. 6, we show integrated intensity (zeroth moment) maps of outflow-tracing molecules $\left({ }^{12} \mathrm{CO}\right.$ and $\left.{ }^{13} \mathrm{CO}\right)$ for the redshifted and blueshifted gas. The minimum intensity below which a pixel is not considered in the creation of the moment maps is based on $5 \sigma \mathrm{rms}$ noise level in emission-free channels. The single-dish $\mathrm{CO}(2-1)$ map (see top left panel of Fig. 6) shows the existence of a bipolar outflow in the overall vicinity of $\mathrm{W} 3\left(\mathrm{H}_{2} \mathrm{O}\right)$ but also encompassing $\mathrm{W} 3(\mathrm{OH})$ in an approximately NE-SW direction. The ${ }^{13} \mathrm{CO}(2-1)$ single-dish map (see top right panel of Fig. 6) shows less extended emission than the ${ }^{12} \mathrm{CO}$ as the ${ }^{13} \mathrm{C}$ isotopologue is a factor of $\sim 76$ less abundant (Henkel et al. 1982), and the map highlights the general NE-SW direction of the outflow even better.

The integrated intensity map of ${ }^{12} \mathrm{CO}$ from SMA interferometric data of Zapata et al. (2011) allows for the detection of two bipolar outflows (see bottom left panel of Fig. 6). The outflow emanating from $\mathrm{W} 3\left(\mathrm{H}_{2} \mathrm{O}\right) \mathrm{E}$ has its blueshifted side to the southwest and its redshifted lobe to the northeast, while the second outflow emanating from $\mathrm{W} 3\left(\mathrm{H}_{2} \mathrm{O}\right) \mathrm{W}$ has its blueshifted side extending to the northeast with its redshifted side to the southwest (Zapata et al. 2011). The difference between the position angles of the two outflows (in the plane of the sky) is $25^{\circ}$. Furthermore, the resulting zeroth moment map of ${ }^{13} \mathrm{CO}$ emission from our combined NOEMA and $30 \mathrm{~m}$ single-dish observations, presented in the bottom right panel of Fig. 6, confirms the findings of Zapata et al. (2011) with regards to the directions and origin of the redshifted outflow lobe from $\mathrm{W} 3\left(\mathrm{H}_{2} \mathrm{O}\right) \mathrm{W}$ and the origins of the blueshifted outflow lobe from $\mathrm{W} 3\left(\mathrm{H}_{2} \mathrm{O}\right) \mathrm{E}$. However, we miss much of the emission that is detected in the ${ }^{12} \mathrm{CO}$ SMA interferometric data, mainly due to the lower abundance of the ${ }^{13} \mathrm{C}$ isotopologue, and thus its lower sensitivity to the outflowing gas. The same coloured arrows obtained from Zapata et al. (2011) are redrawn in a zoom panel inside 
W3 $\left(\mathrm{H}_{2} \mathrm{O}\right)$

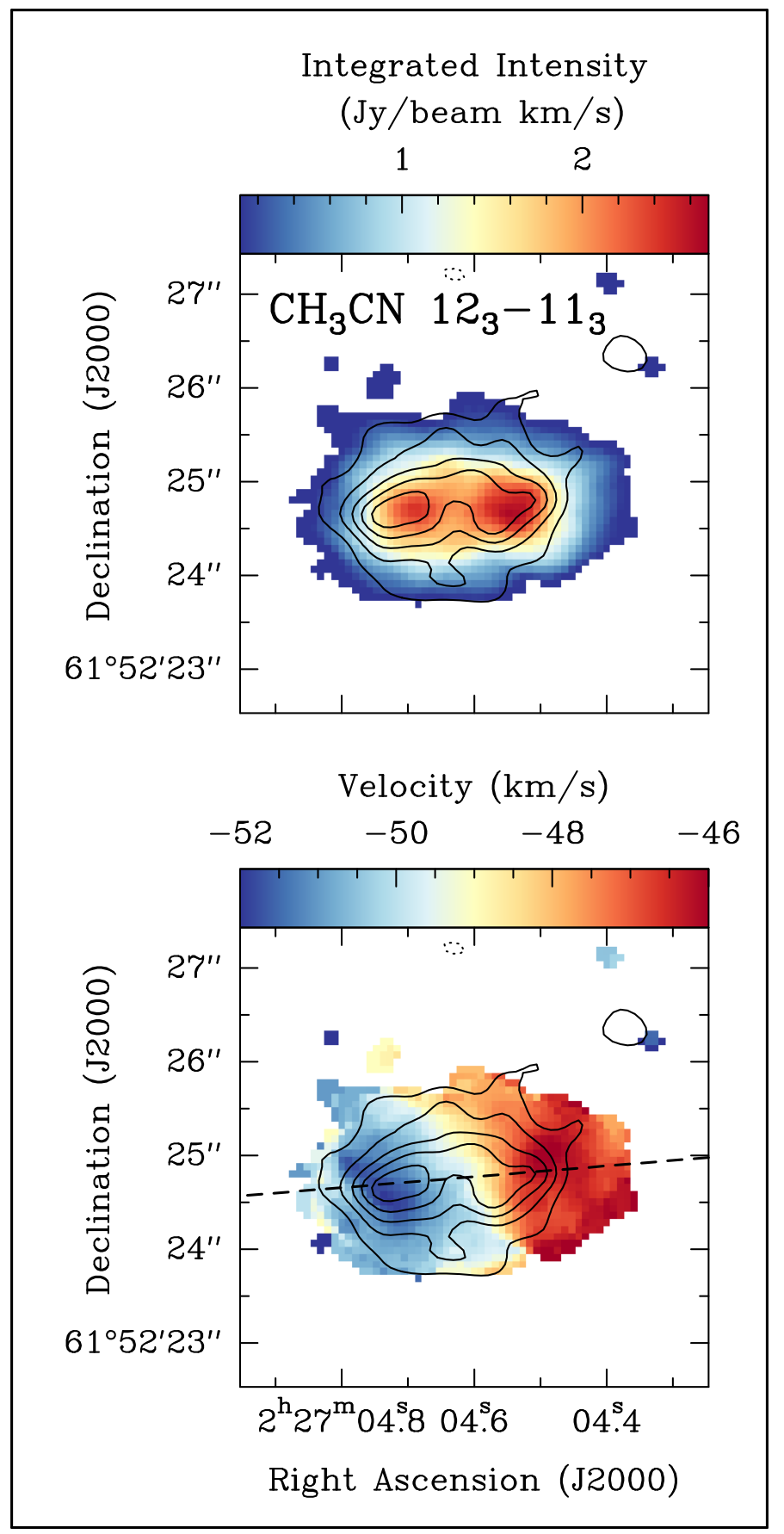

W3(OH)

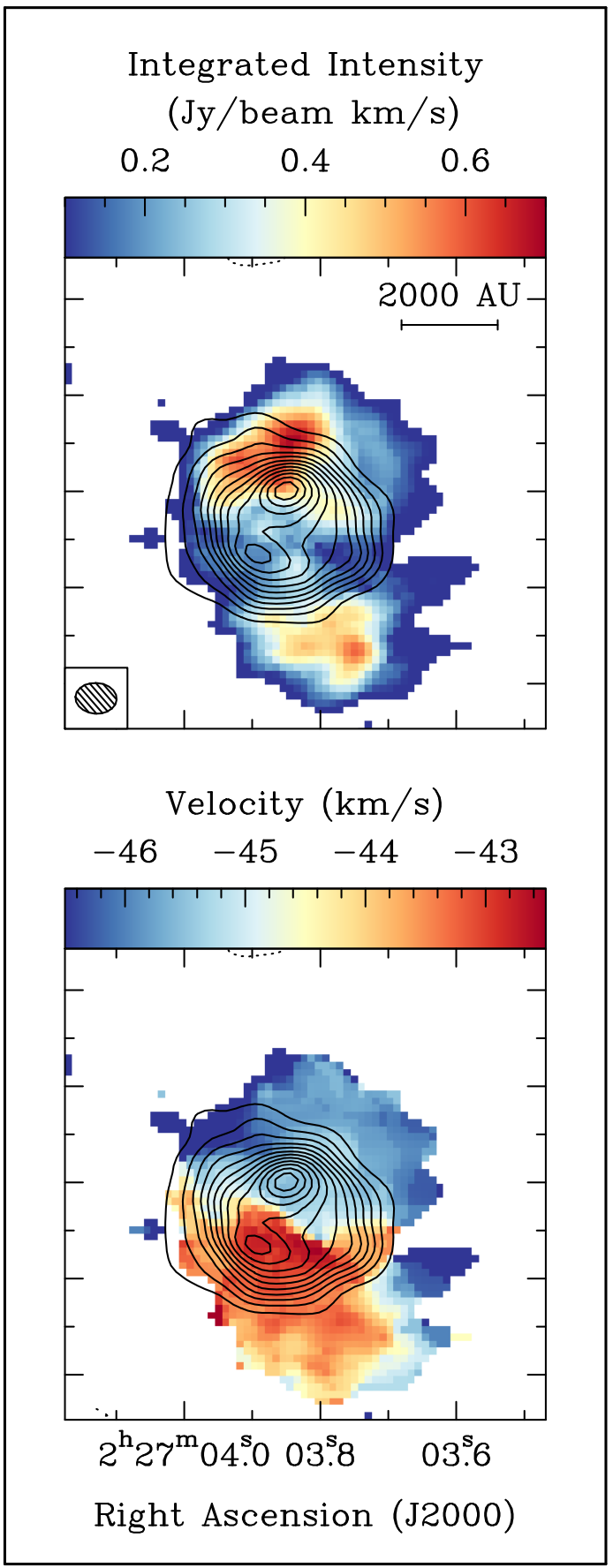

Fig. 5. Top row: integrated intensity (zeroth moment) map of $\mathrm{CH}_{3} \mathrm{CN}\left(12_{3}-11_{3}\right)$ for $\mathrm{W} 3\left(\mathrm{H}_{2} \mathrm{O}\right)$ (left panel) and $\mathrm{W} 3(\mathrm{OH})$ (right panel) in the $\mathrm{ABD}$ configuration. Bottom row: intensity-weighted peak velocity (first moment) map of $\mathrm{CH}_{3} \mathrm{CN}\left(12_{3}-11_{3}\right)$ for $\mathrm{W} 3\left(\mathrm{H}_{2} \mathrm{O}\right)($ left panel $)$ and W3(OH) (right panel) in the ABD configuration. The dashed line corresponds to the cut made for the PV plot of $\mathrm{W} 3\left(\mathrm{H}_{2} \mathrm{O}\right)$ presented in Fig. 8. The solid contours correspond to the $1.37 \mathrm{~mm}$ continuum, starting at $6 \sigma$ and increasing in steps of $6 \sigma\left(1 \sigma=3.2 \mathrm{mJy}\right.$ beam $\left.^{-1}\right)$. The dotted contours correspond to the same negative levels. A scale bar and the synthesised beam $\left(00^{\prime} 43 \times 00^{\prime} 32, \mathrm{PA}=86^{\circ}\right)$ are shown in the top right panel.

the bottom right panel of this figure, highlighting that the two outflows are in fact emanating from different positions.

In Fig. 7, we show how the centimetre emission aligns with the millimetre continuum emission. The directions of bipolar molecular outflows emanating from the cores are shown by red and blue arrows, and the positions of water masers shown by yellow triangles. The elongated radio source centred on
$\mathrm{W} 3\left(\mathrm{H}_{2} \mathrm{O}\right) \mathrm{E}$ has a spectral index of -0.6 and is a source of synchrotron emission, best described by a jet-like model due to its morphology (Reid et al. 1995; Wilner et al. 1999). The radio source centred on $\mathrm{W} 3\left(\mathrm{H}_{2} \mathrm{O}\right) \mathrm{W}$, however, has a rising spectral index, possibly due to a circumstellar wind being ionised by an embedded (proto)stellar source at this position. Furthermore, the discrepancy between the direction of the synchrotron jet-like 

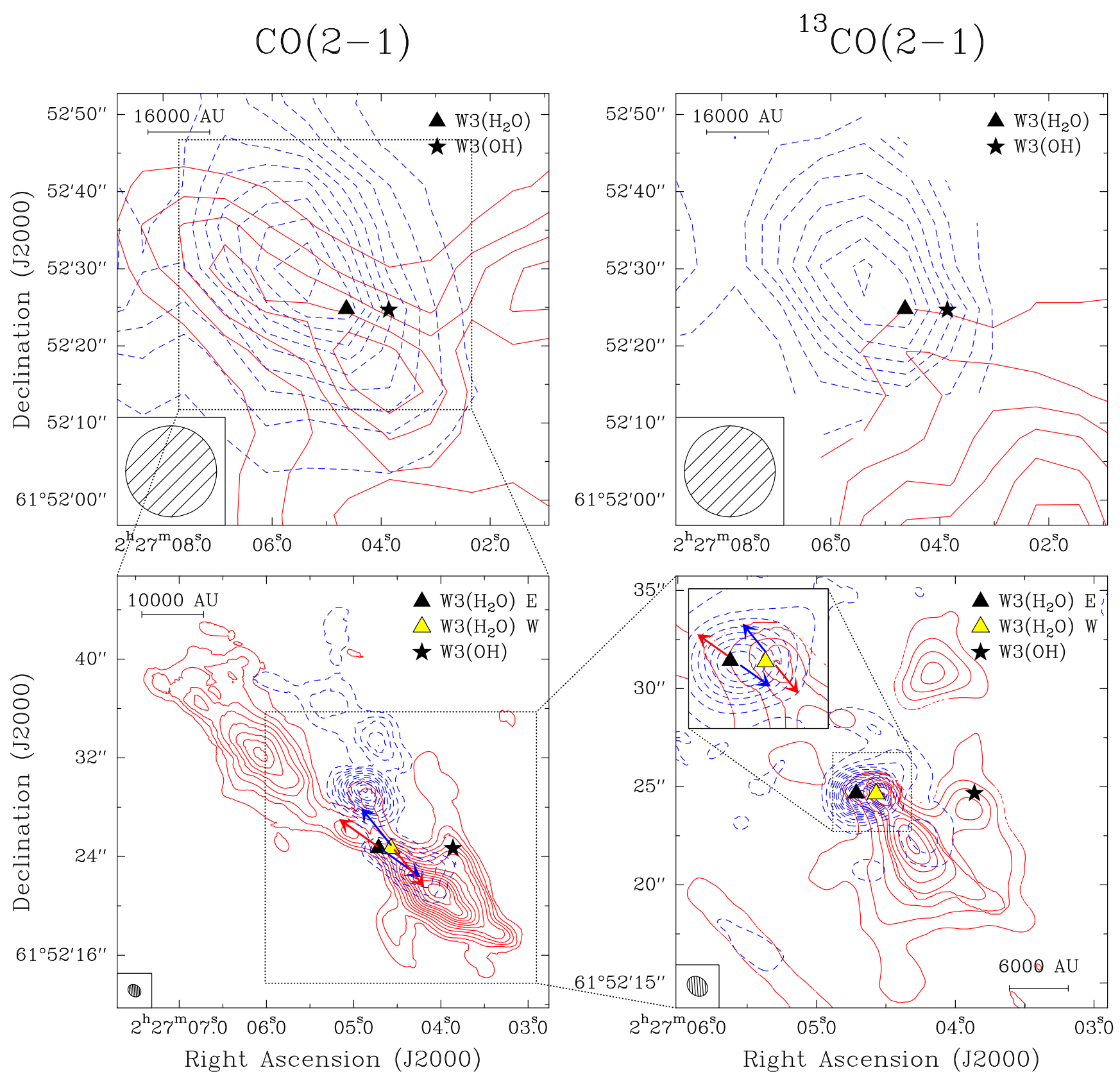

Fig. 6. Top left panel: intensity map of $\mathrm{CO}(2-1)$ emission from IRAM $30 \mathrm{~m}$ integrated over the velocity range from -40 to $-20 \mathrm{~km} \mathrm{~s}{ }^{-1}$ for the redshifted and from -80 to $-55 \mathrm{~km} \mathrm{~s}^{-1}$ for the blueshifted gas. Contours start at $5 \sigma$ and increase in steps of $5 \sigma\left(1 \sigma=1.9 \mathrm{~K} \mathrm{~km} \mathrm{~s}{ }^{-1}\right)$. Top right panel: intensity map of ${ }^{13} \mathrm{CO}(2-1)$ emission from IRAM $30 \mathrm{~m}$ integrated over the velocity range from -40 to $-20 \mathrm{~km} \mathrm{~s}^{-1}$ for the redshifted and from -80 to $-55 \mathrm{~km} \mathrm{~s}^{-1}$ for the blueshifted gas. Contours start at $3 \sigma$ and increase in steps of $2 \sigma\left(1 \sigma=0.42 \mathrm{~K} \mathrm{~km} \mathrm{~s}^{-1}\right)$. Bottom left panel: intensity map of $\mathrm{CO}(2-1)$ emission obtained by Zapata et al. (2011) with the SMA integrated over the velocity range from -45 to -25 for the redshifted and from -75 to -55 for the blueshifted gas. Contours start at $5 \sigma$ and increase in steps of $5 \sigma\left(1 \sigma=1.1 \mathrm{mJy} \mathrm{beam}^{-1} \mathrm{~km} \mathrm{~s}^{-1}\right)$. The blue and red arrows highlight the positions and directions of the $\mathrm{CO}$ outflows emanating from each source. Bottom right panel: intensity map of ${ }^{13} \mathrm{CO}(2-1)$ emission from IRAM 30 m merged with NOEMA observations integrated over the velocity range from -41 to -30 for the redshifted and from -70 to -53 for the blueshifted gas. Contours start at $3 \sigma$ and increase in steps of $3 \sigma\left(1 \sigma=0.03\right.$ mJy beam ${ }^{-1} \mathrm{~km} \mathrm{~s}^{-1}$ for redshifted and $0.17 \mathrm{mJy}$ beam ${ }^{-1}$ $\mathrm{km} \mathrm{s}^{-1}$ for blueshifted sides). The zoom panel in the top left corner highlights the launching positions of each outflow, with the red and blue arrows showing the directions of the two bipolar outflows from Zapata et al. (2011). The rms noise used in drawing the contours of the integrated intensity maps have been determined by first creating the maps without any constraints on the minimum emission level (threshold of 0) and calculating the noise in an emission-free part of the resulting map.

object and the molecular outflow could be due to the existence of multiple objects in this core, unresolved by our observations. Interestingly, it has been shown that synchrotron radiation can be produced not only via jets, but also through the acceleration of relativistic electrons in the interaction of disk material with a stellar wind (Shchekinov \& Sobolev 2004), providing an alternative explanation for the maser and $\mathrm{cm}$ emission.

\section{Analysis and discussion}

\subsection{Dense gas kinematics}

The kinematics of $\mathrm{W} 3\left(\mathrm{H}_{2} \mathrm{O}\right)$ can be further analysed by looking at position-velocity (PV) diagrams for various transitions of dense gas and potentially disk-tracing molecules such as 

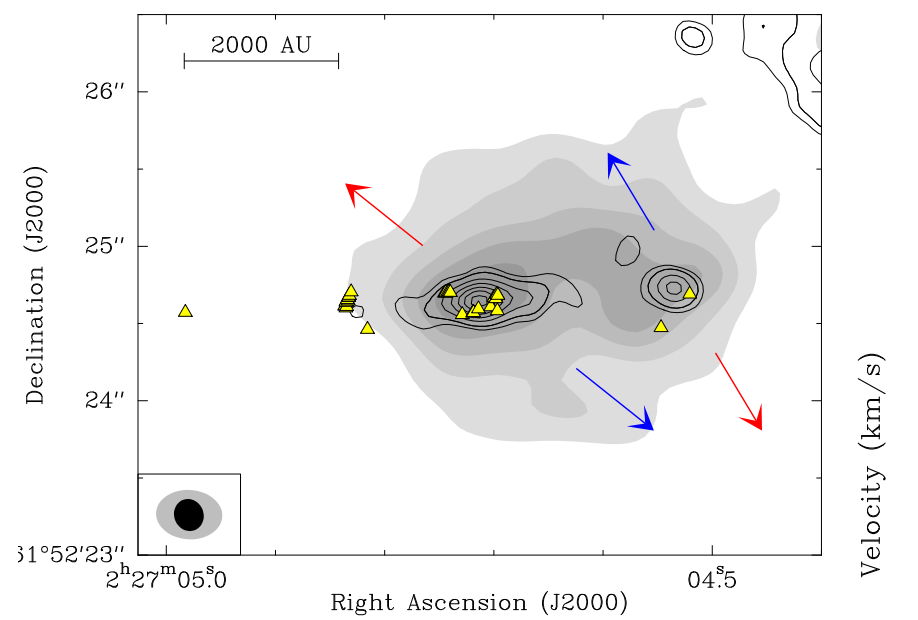

Fig. 7. NOEMA $1.37 \mathrm{~mm}(219 \mathrm{GHz})$ continuum image toward $\mathrm{W} 3\left(\mathrm{H}_{2} \mathrm{O}\right)$ in grey with levels starting at $6 \sigma$ and increasing in steps of $6 \sigma$. The black contours correspond to the $\mathrm{cm}$ emission from Wilner et al. (1999). The positions of $\mathrm{H}_{2} \mathrm{O}$ masers obtained from Hachisuka et al. (2006) are plotted as yellow triangles. The blue and red arrows show the directions of bipolar molecular outflows from Zapata et al. (2011; see Fig. 6). The synthesised beam size of the $\mathrm{cm}$ emission $\left(00^{\prime} 21 \times 00^{\prime} 19, \mathrm{PA}=68^{\circ}\right)$ is shown in black in the bottom left corner. The synthesised beam size of our mm continuum image is shown in grey in the bottom left corner.

$\mathrm{CH}_{3} \mathrm{CN}$ and $\mathrm{HCOOCH}_{3}$ (e.g. Beuther et al. 2005). In the following, we divide our focus between the large-scale kinematics of the entire $\mathrm{W} 3\left(\mathrm{H}_{2} \mathrm{O}\right)$ region, where we put forward arguments for the observed velocity gradients in $\mathrm{CH}_{3} \mathrm{CN}$ and $\mathrm{HCOOCH}_{3}$ being due to rotation instead of infall (Sect. 4.1.1), and the small-scale kinematics of the two separate cores within $\mathrm{W} 3\left(\mathrm{H}_{2} \mathrm{O}\right)$ (Sect. 4.1.2). Although the alignment of the elongated radio emission with the water masers can be described by an outflow model oriented in the east-west direction, the detection of two molecular outflows emanating from the positions of the two continuum peaks, roughly perpendicular to the observed velocity gradient in $\mathrm{CH}_{3} \mathrm{CN}$ at large scale and perpendicular to the observed velocity gradients on smaller scales (see Sect. 4.1.2), make it unlikely that the motions in $\mathrm{CH}_{3} \mathrm{CN}$ would be due to expansion or outflow. Furthermore, $\mathrm{CH}_{3} \mathrm{CN}$ and $\mathrm{HCOOCH}_{3}$ line profiles do not show the broad components typically seen in emission from expanding gas, and these species are nevertheless too complex to exist in an ionised jet. We, therefore, conclude that the observed velocity gradient is most likely due to rotation, which we will assume for the remainder of this paper.

\subsubsection{Large-scale kinematics}

The PV plots of $\mathrm{CH}_{3} \mathrm{CN}$ and $\mathrm{HCOOCH}_{3}$ for $\mathrm{W} 3\left(\mathrm{H}_{2} \mathrm{O}\right)$ are shown in Fig. 8 for a cut in the direction of the velocity gradient going through the continuum peaks (dashed line in bottom left panel of Fig. 5) obtained from the NOEMA observations. The white curves in the top left panel correspond to gas in Keplerian rotation with $V_{\text {rot }} \propto R^{-1 / 2}$ about a 10,25 , and $50 M_{\odot}$ central object. These white curves are not fits to the PV diagram, but are merely drawn to guide the eye. It is clear that the gas is not in Keplerian rotation; however, higher velocity gas is observed closer to the centre of $\mathrm{W} 3\left(\mathrm{H}_{2} \mathrm{O}\right)$ which can be a signpost for differential rotation.

Ohashi et al. (1997) created models for comparison to their interferometric data of the low-mass protostar L1527, using a

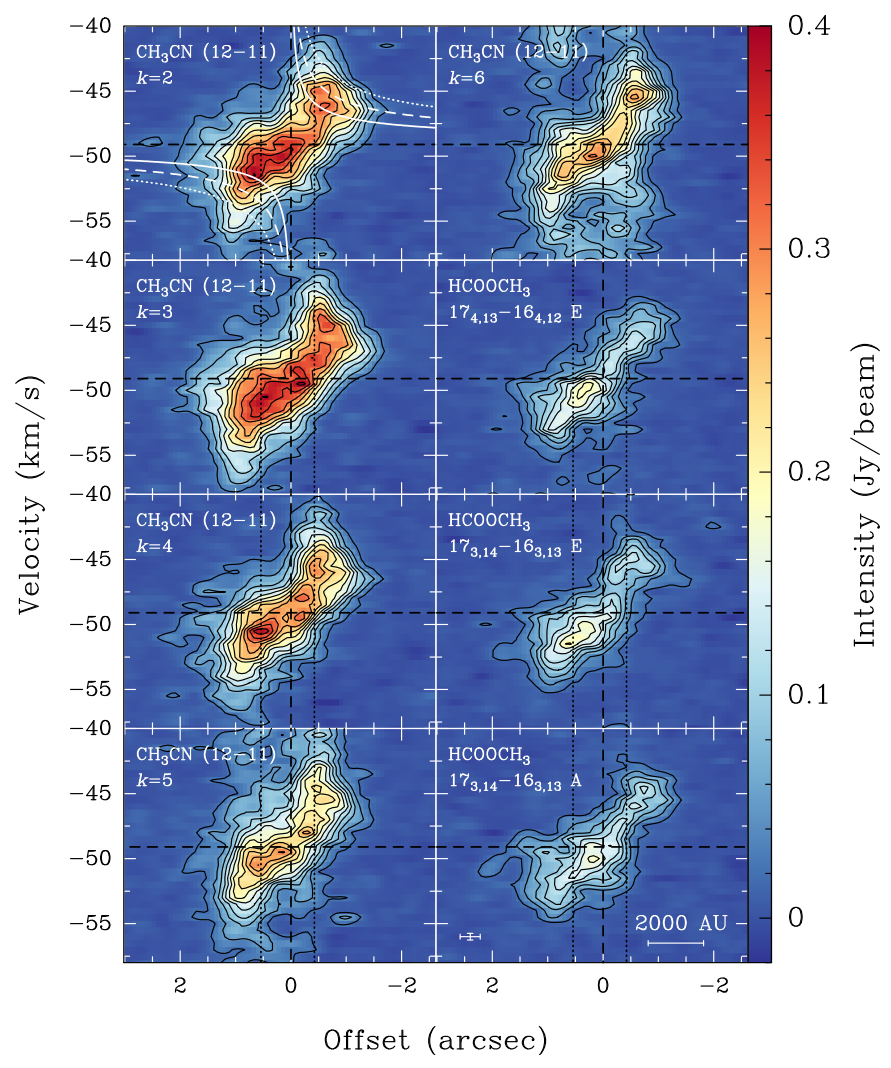

Fig. 8. Position-velocity plots of $\mathrm{W} 3\left(\mathrm{H}_{2} \mathrm{O}\right)$ for a cut in the direction of rotation as depicted by a dashed line in the bottom left panel of Fig. 5 for various species and transitions in the ABD configuration. The vertical dashed lines correspond to the centre of the cut. The vertical dotted lines correspond to the positions of continuum peaks corresponding to $\mathrm{W} 3\left(\mathrm{H}_{2} \mathrm{O}\right) \mathrm{E}$ and $\mathrm{W} 3\left(\mathrm{H}_{2} \mathrm{O}\right) \mathrm{W}$. The horizontal dashed lines correspond to the LSR velocity of $\mathrm{W} 3\left(\mathrm{H}_{2} \mathrm{O}\right)$. The black contours start at $4 \sigma$ and increase in steps of $6 \sigma$. The white solid, dashed, and dotted lines in the top left panel correspond to the region within which emission is expected if the gas is in a disk in Keplerian rotation about a 10, 25, $50 M_{\odot}$ star, respectively. The white curves are not fits to the rotation curve, but are drawn to guide the eye. A scale bar and a cross that corresponds to the spatial and spectral resolutions are shown in the bottom right panel.

thin disk with 2000 AU extent configured edge-on and present PV diagrams for cases with various degrees of infall and rotation. In the case of infall-only motions, their PV plots are axisymmetric with two peaks offset symmetrically in the velocity axis. With the addition of rotation, the peaks become blueshifted and redshifted away from the central positions such that in the case of a pure Keplerian rotation and in the absence of infall we would recover the classical butterfly-shaped rotation curve. Comparing our PV plots to the Ohashi et al. (1997) scenarios, we observe minimal contributions in the top left and bottom right quadrants of our plots where one would expect some emission in the cases including infall motions. Therefore, we do not detect infall motions in our interferometric data probably because the infalling envelope is too diffuse and filtered out. Furthermore, models by Tobin et al. (2012) for spherical rotating collapse and filamentary rotating collapse showed similar results to that of Ohashi et al. (1997), confirming that the absence of infall results in the lack of emission in those quadrants and not the projection or source morphology. Moreover, the PV plots of $\mathrm{HCOOCH}_{3}$, which is a less abundant species than $\mathrm{CH}_{3} \mathrm{CN}$, are more representative of rigid body-like rotation. 
Chen et al. (2006) generated a binary model with radiative transfer post-processing of methyl cyanide for each source within $\mathrm{W} 3\left(\mathrm{H}_{2} \mathrm{O}\right)$, showing that the high-velocity deviations from solid body rotation in their PV plots could be a result of two spatially unresolved cores (on similar scales to our observations) with a small radial velocity difference. The detection of a smooth velocity gradient in all tracers across $\mathrm{W} 3\left(\mathrm{H}_{2} \mathrm{O}\right) \mathrm{E}$ and $\mathrm{W}$ suggests that the observed angular velocity difference cannot be solely due to binary motion of the cores, but that there is also a large-scale toroidal structure encompassing and rotating about the two resolved cores. Such circumbinary toroidal structures have indeed been observed previously at lower angular resolutions in other sources (e.g. Beltrán et al. 2005; Beuther et al. 2007).

\subsubsection{Small-scale kinematics}

Although we see a smooth velocity gradient in $\mathrm{CH}_{3} \mathrm{CN}$ across the entire $\mathrm{W} 3\left(\mathrm{H}_{2} \mathrm{O}\right)$ structure, the existence of at least two cores within it as presented by our continuum results, and two collimated outflows requires further analysis. Imaging the data in the most extended configuration and therefore reaching our highest resolution, we can filter out the large-scale envelope to analyse the kinematics of gas around each of the cores. Figure 9 shows the first moment map of $\mathrm{CH}_{3} \mathrm{CN}\left(12_{3}-11_{3}\right)$ for the cores to the east and west using the A-array observations exclusively. The maps have been scaled and masked to highlight the velocity structure of each core. The mean line-of-sight velocity difference between the two main cores, $\mathrm{W} 3\left(\mathrm{H}_{2} \mathrm{O}\right) \mathrm{W}$ and $\mathrm{E}$, is a few $\mathrm{km} \mathrm{s}^{-1}$. Velocity differences of a few $\mathrm{km} \mathrm{s}^{-1}$ are observed across each core, approximately perpendicular to the directions of the bipolar molecular outflows emanating from each core (Zapata et al. 2011). This indicates that the small-scale gradients across each individual core are most likely due to rotation. The two line-of-sight velocity gradients are on the order of $\sim 1000 \mathrm{~km} \mathrm{~s}^{-1} \mathrm{pc}^{-1}$, depending on the choice for the extent of the gradient, much faster than the rotational motion of $170 \mathrm{~km} \mathrm{~s}^{-1} \mathrm{pc}^{-1}$ for the whole of $\mathrm{W} 3\left(\mathrm{H}_{2} \mathrm{O}\right)$ from the ABD data. Furthermore, the directions of the velocity gradients observed for each core are slightly inclined with respect to the overall east-west motion of the gas on larger scales (Fig. 5), suggesting that the general rotation seen around the two cores may be inherited from the large-scale rotation. Meanwhile, the direction of the blueshifted (redshifted) outflow emanating from $\mathrm{W} 3\left(\mathrm{H}_{2} \mathrm{O}\right) \mathrm{W}$ is almost in the opposite direction of the blueshifted (redshifted) outflow ejected from $\mathrm{W} 3\left(\mathrm{H}_{2} \mathrm{O}\right) \mathrm{E}$. This implies that the inclination angles of the two rotating structures with respect to the plane of the sky are likely different.

Figure 10 shows PV diagrams for $\mathrm{W} 3\left(\mathrm{H}_{2} \mathrm{O}\right) \mathrm{E}$ (left) and $\mathrm{W} 3\left(\mathrm{H}_{2} \mathrm{O}\right) \mathrm{W}$ (right) corresponding to cuts in the directions of rotation as depicted by dashed lines in Fig. 9. Based on the PV plots, the LSR velocities of $\mathrm{W} 3\left(\mathrm{H}_{2} \mathrm{O}\right) \mathrm{E}$ and $\mathrm{W} 3\left(\mathrm{H}_{2} \mathrm{O}\right) \mathrm{W}$ are estimated to be -51 and $-47 \mathrm{~km} \mathrm{~s}^{-1}$, respectively. White curves correspond to gas in Keplerian rotation about a 5, 10, and $15 M_{\odot}$ centrally dominated object. As on larger scales, these PV diagrams do not show the symmetric four-quadrant shape expected if the velocity gradients were due to infall.

The PV plot for $\mathrm{W} 3\left(\mathrm{H}_{2} \mathrm{O}\right) \mathrm{W}$ contains contributions from $\mathrm{W} 3\left(\mathrm{H}_{2} \mathrm{O}\right) \mathrm{E}$ due to the angle and extent of the cut, hence there is added emission in quadrants toward positive offset, making it difficult to infer rotational signatures pertaining to the blueshifted emission of $\mathrm{W} 3\left(\mathrm{H}_{2} \mathrm{O}\right) \mathrm{W}$. The redshifted rotational signatures seen in the quadrants toward negative offsets,

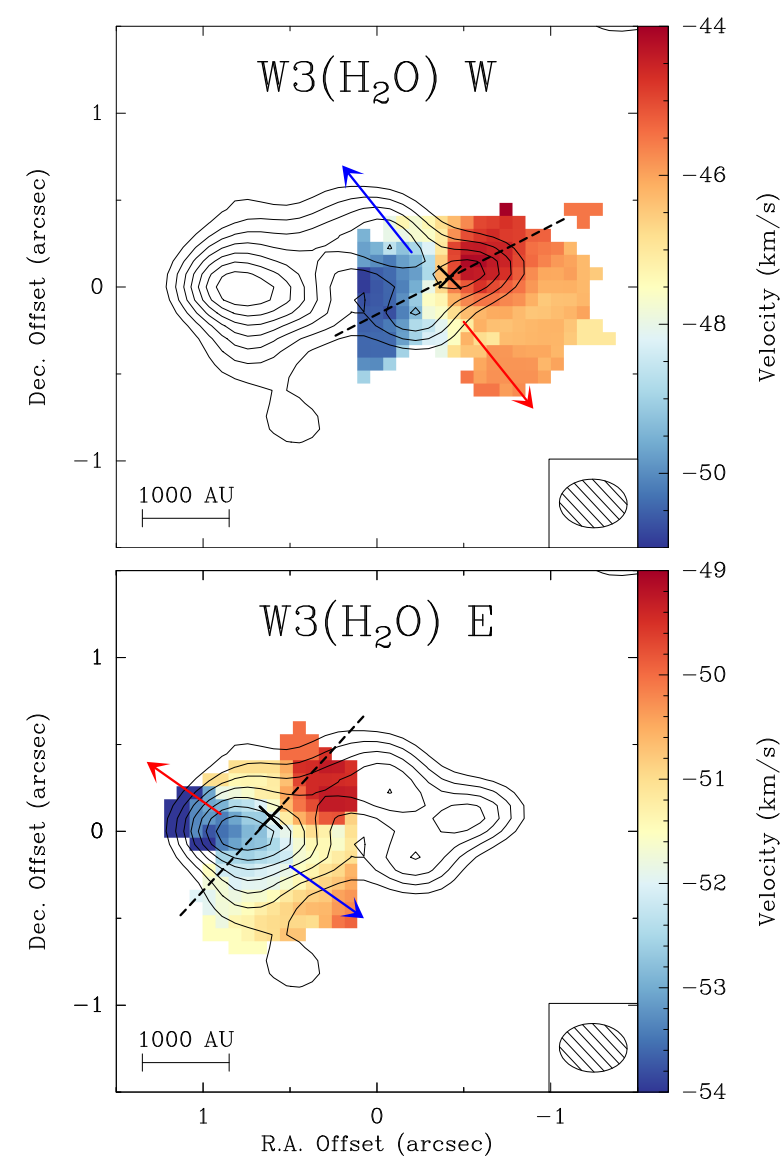

Fig. 9. Intensity-weighted peak velocity (first moment) map of $\mathrm{CH}_{3} \mathrm{CN}\left(12_{3}-11_{3}\right)$ using only the A-array observations and masked out to show contributions from $\mathrm{W} 3\left(\mathrm{H}_{2} \mathrm{O}\right) \mathrm{W}$ (top panel) and $\mathrm{W} 3\left(\mathrm{H}_{2} \mathrm{O}\right) \mathrm{E}$ (bottom panel). The solid contours correspond to the $1.37 \mathrm{~mm}$ continuum in the A-array only observations and start at $6 \sigma$ and increase in steps of $3 \sigma\left(1 \sigma=2.5 \mathrm{mJy}\right.$ beam $\left.^{-1}\right)$. The dashed lines correspond to the cuts made for the PV plots (Fig. 10). The blue and red arrows show the directions of bipolar molecular outflows (Fig. 6). A scale bar and the synthesised beam $\left(0{ }^{\prime} 39 \times 00^{\prime} .28, \mathrm{PA}=88^{\circ}\right)$ are shown in the bottom. The velocity ranges are different for the two cores.

however, show signatures of increased gas velocities closer to the centre of the core. Such a trend in the PV plot implies differential rotation of material, possibly within a disk-like object.

Position-velocity plots for $\mathrm{W} 3\left(\mathrm{H}_{2} \mathrm{O}\right)$ E have a lower $\mathrm{S} / \mathrm{N}$ than $\mathrm{W} 3\left(\mathrm{H}_{2} \mathrm{O}\right) \mathrm{W}$ as line emission is typically weaker for this fragment even though its $1.37 \mathrm{~mm}$ dust continuum peak is stronger. The linearity of the rotation curves in $\mathrm{CH}_{3} \mathrm{CN}$ does not reveal Keplerian signatures, but is more consistent with rigid bodylike rotation. In order to increase the $\mathrm{S} / \mathrm{N}$, we stacked the PV plots of $\mathrm{CH}_{3} \mathrm{CN}\left(12_{K}-11_{K}\right), K=2-5$ and show this stacked PV plot in the bottom right panel of Fig. 10 for $\mathrm{W} 3\left(\mathrm{H}_{2} \mathrm{O}\right) \mathrm{E}$. Stacking is a reasonable strategy as the variation in average linewidths of these transitions around this core is below our spectral resolution; therefore, assuming these lines to be probing the same surface is valid. In the stacked image, the $4 \sigma$ contour reveals a high-velocity feature close to the centre of the core toward positive offsets. As this feature has an extent of the order of our spatial resolution, it is unclear whether the rotation observed for $\mathrm{W} 3\left(\mathrm{H}_{2} \mathrm{O}\right) \mathrm{E}$ is due to a disk-like object, an unresolved binary (or multiple) system, or a combination of the two. 

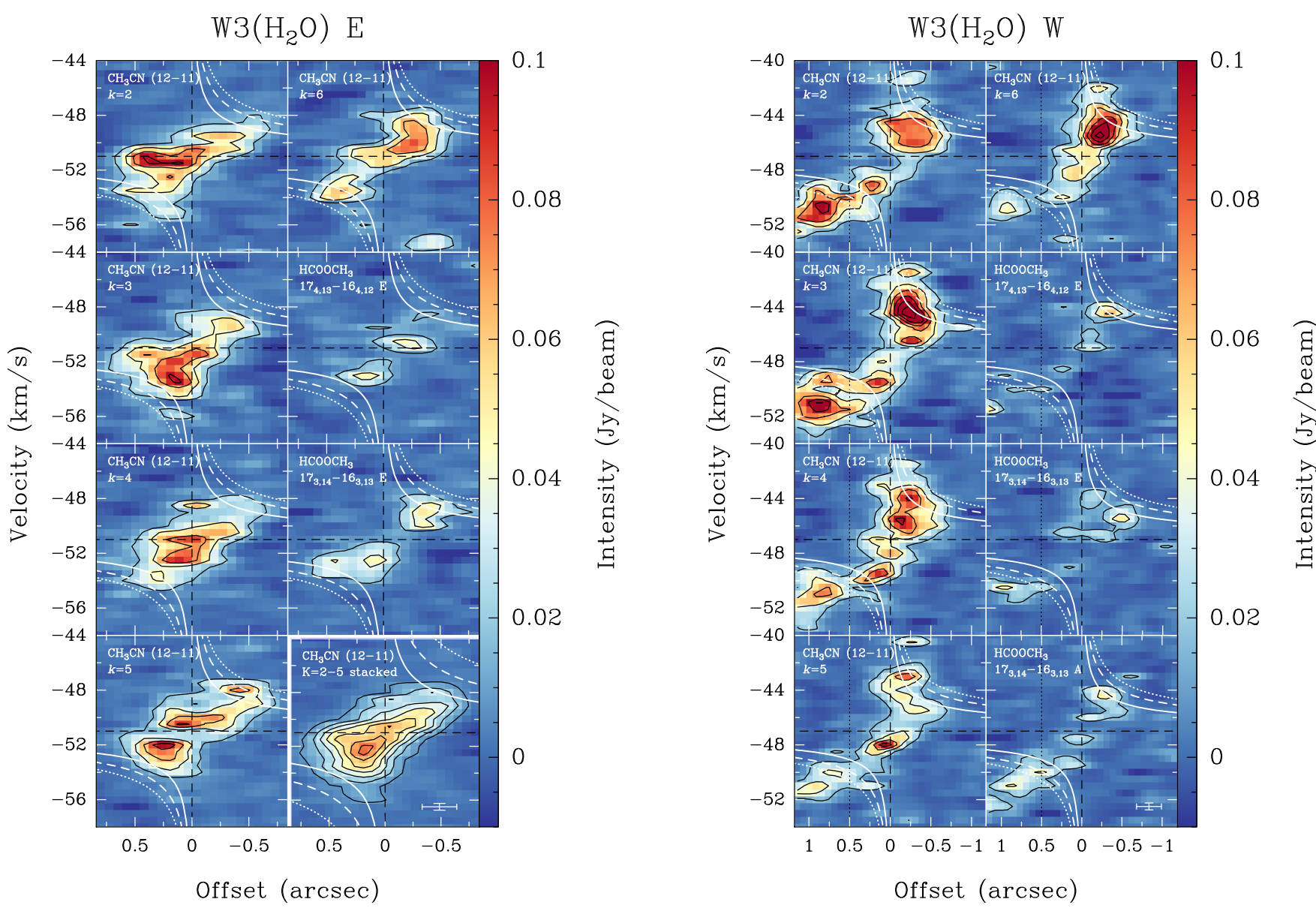

Fig. 10. Position-velocity plots along a cut in the direction of rotation as depicted by dashed lines in Fig. 9 for a fragment to the east (left panel) and to the west (right panel). The black contours start at $4 \sigma$ and increase in steps of $6 \sigma$. The white solid, dashed, and dotted lines correspond to the region within which emission is expected if the gas is in a disk in Keplerian rotation about a 5, 10, and $15 M_{\odot}$ star, respectively. The crosses in the bottom right corners correspond to the spatial and spectral resolutions. Regions to the left of the dotted vertical line in the right figure contain contributions from $\mathrm{W} 3\left(\mathrm{H}_{2} \mathrm{O}\right) \mathrm{E}$.

\subsection{Temperature distribution}

As a symmetric top molecule, $\mathrm{CH}_{3} \mathrm{CN}$ is an excellent thermometer of hot molecular gas (e.g. Loren \& Mundy 1984; Zhang et al. 1998) since its relative populations in different $K$-levels are dominated by collisions. Our high spectral resolution observations of $\mathrm{CH}_{3} \mathrm{CN}$ covers its $J=12-11, K=0-6$ transitions and some of their isotopologues which have upper energy levels in the range $70-325 \mathrm{~K}$ with $0.5 \mathrm{~km} \mathrm{~s}^{-1}$ spectral resolution (see Table 3).

We made use of the eXtended CASA Line Analysis Software Suite (XCLASS ${ }^{4}$; Möller et al. 2017) to model the observed spectra under the assumption of Local Thermodynamical Equilibrium (LTE), which is typically valid for $\mathrm{CH}_{3} \mathrm{CN}$ in such high-density environments (see Sect. 4.3). In summary, XCLASS solves the radiative transfer equation in one dimension for a set of initial conditions (source size, column density, temperature, linewidth, and peak velocity) provided by the user, and through a $\chi^{2}$ minimisation routine changes the initial conditions within ranges that have been provided by the user to obtain the best fit to the observed spectra. The details of our XCLASS modelling are summarised in Appendix B.

In Fig. 11, we present our results of pixel-by-pixel XCLASS modelling of $\mathrm{CH}_{3} \mathrm{CN}\left(12_{K}-11_{K}\right), K=4-6$, including

4 https://xclass.astro. uni-koeln.de
$\mathrm{CH}_{3}{ }^{13} \mathrm{CN}\left(12_{K}-11_{K}\right), K=0-3$, in AB configuration which produces rotational temperature, column density, peak velocity, and linewidth maps for $\mathrm{CH}_{3} \mathrm{CN}$. The column density map is double peaked, similar to the continuum emission, although the column density peaks are slightly offset to the northwest by a synthesised beam. This offset is consistent with the offset found between the continuum peaks and the integrated intensity maps of $\mathrm{CH}_{3} \mathrm{CN}\left(12_{3}-11_{3}\right.$; see Fig. 5) and most high-density tracers (see Fig. A.1). The median $\mathrm{CH}_{3} \mathrm{CN}$ column density is $1.4 \times 10^{15} \mathrm{~cm}^{-2}$. The velocity gradient derived in this way is consistent with the large-scale east-west velocity gradient observed in the first moment map of $\mathrm{CH}_{3} \mathrm{CN}$, confirming that our modelling strategy captures this accurately, regardless of its origin. The linewidths are also larger by a few $\mathrm{km} \mathrm{s}^{-1}$ for $\mathrm{W} 3\left(\mathrm{H}_{2} \mathrm{O}\right) \mathrm{W}$, also seen in the second moment maps. The median rotational temperature of $\mathrm{W} 3\left(\mathrm{H}_{2} \mathrm{O}\right)$ is $\sim 165 \mathrm{~K}$ and the temperature structure does not follow any particular pattern within either core. Two high-temperature features can be seen which may be associated with regions carved out by the molecular outflows allowing a deeper look into the cores, or regions that may have been additionally heated by the outflow. Nevertheless, the compactness of these features, which are of the same order as the size of our synthesised beam, along with decreased S/N at the edges of the map, prevent us from making firm conclusions in this regard. 

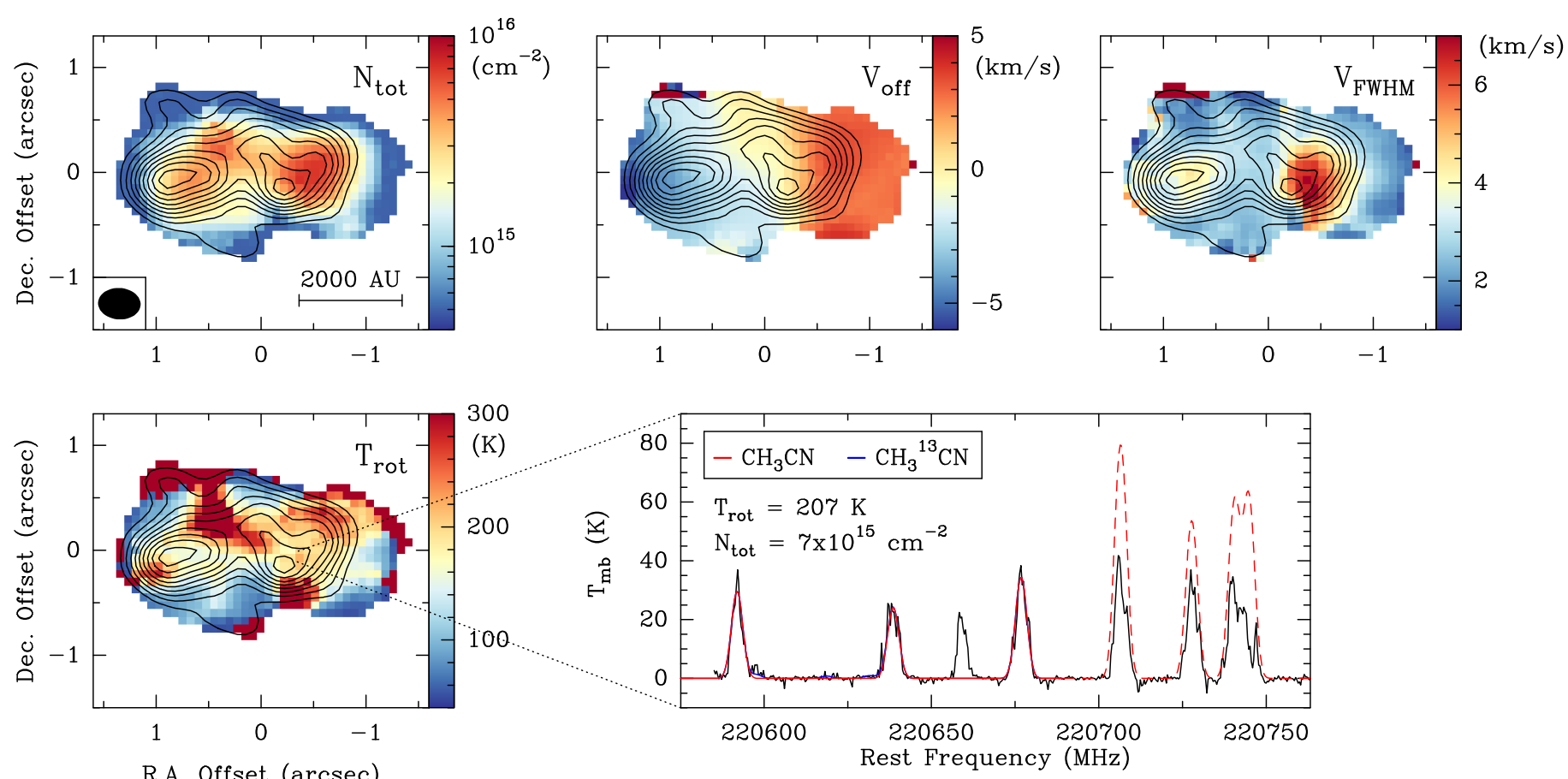

Fig. 11. Column density (top left panel), velocity offset (top middle panel), full width at half maximum linewidth (top right panel), and rotational temperature (bottom left panel) maps obtained by fitting $\mathrm{CH}_{3} \mathrm{CN}\left(12_{K}-11_{K}\right), K=4-6$ and $\mathrm{CH}_{3}{ }^{13} \mathrm{CN}\left(12_{K}-11_{K}\right), K=0-3$ lines simultaneously with XCLASS. The black contours correspond to the continuum image in the AB configuration, start at $6 \sigma$ and increase in steps of $3 \sigma$ (see Table 4 ). Bottom right panel: observed spectrum of a given pixel drawn in black and overlaid with the resulting fit for $\mathrm{CH}_{3} \mathrm{CN}\left(12_{K}-11_{K}\right), K=4-6$ in red and the marginally detected $\mathrm{CH}_{3}{ }^{13} \mathrm{CN}\left(12_{K}-11_{K}\right), K=0-3$ in blue. The dashed red line corresponds to the predicted fit for the $\mathrm{CH}_{3} \mathrm{CN}$ lines that were not used in the fitting process (see explanation in Appendix B). The bright line detected between $K=4$ and 5 components is identified as $\mathrm{C}_{2} \mathrm{H}_{5} \mathrm{CN}$. The corresponding fit parameters are provided in the panel. Regions outside of the most extended combination of $6 \sigma$ contours of integrated intensity of $\mathrm{CH}_{3} \mathrm{CN}$ lines are masked out.

\subsection{Mass estimates}

The combined bolometric luminosity of $\mathrm{W} 3\left(\mathrm{H}_{2} \mathrm{O}\right)$ and $\mathrm{W} 3(\mathrm{OH})$, determined from fitting the SED from the near-IR to sub-mm, is $8.3 \times 10^{4} L_{\odot}$ (Mottram et al. 2011). The contribution from $\mathrm{OH}$ can be estimated by first calculating the corresponding flux of ionising photons (see e.g. Appendix B.2 of Sánchez-Monge 2011, for more details) using

$\left(\frac{Q_{0}}{\text { photons s}^{-1}}\right)=8.852 \times 10^{40}\left(\frac{F_{v}}{\mathrm{Jy}}\right)\left(\frac{v}{\mathrm{GHz}}\right)^{0.1}\left(\frac{T_{\mathrm{e}}}{\mathrm{K}}\right)^{0.35}\left(\frac{d}{\mathrm{pc}}\right)^{2}$,

where $F_{v}$ is the flux density of the free-free radio continuum emission at frequency $v, T_{\mathrm{e}}$ is the electron temperature, and $d$ is the distance. The observed integrated radio flux at $15 \mathrm{GHz}$ for $\mathrm{W} 3(\mathrm{OH})$ is $2.53 \mathrm{Jy}$ (Kurtz et al. 1994), and assuming a typical electron temperature of $10^{4} \mathrm{~K}$, this results in a value of $Q_{0}=1.2 \times 10^{48}$ photons s$^{-1}$. Interpolating from Table 1 of Davies et al. (2011) and the relationships between spectral type and photospheric temperature from Martins et al. (2005) and Boehm-Vitense (1981) for O and B stars, respectively, this ionising photon flux approximately corresponds to an O8.5 main sequence star, with $M \approx 20 M_{\odot}$ and $L \approx 4.4 \times 10^{4} L_{\odot}$. This leaves a total bolometric luminosity of $3.9 \times 10^{4} L_{\odot}$, which we assume to be evenly distributed between the two cores within $\mathrm{W} 3\left(\mathrm{H}_{2} \mathrm{O}\right)$, $1.95 \times 10^{4} L_{\odot}$ each; using the same tables this would correspond to two $15 M_{\odot}$ stars of spectral type B0.

The above estimates are based on Davies et al. (2011) for zero age main sequence (ZAMS) stars. High-mass protostars growing by accretion resemble ZAMS stars in terms of their luminosities and temperatures when core nuclear burning dominates other sources of luminosity such as accretion and envelope burning. When this occurs depends primarily on when the protostar gains sufficient mass, but also on the accretion rate. Stellar structure calculations suggest that this occurs at 5-10 $M_{\odot}$ for accretion rates of $10^{-5}-10^{-4} M_{\odot} \mathrm{yr}^{-1}$, respectively (Norberg \& Maeder 2000; Behrend \& Maeder 2001; Keto \& Wood 2006). The mass estimates further assume that all the emitted energy has been produced within the (proto)stars, ignoring contributions from episodic accretion to the luminosity. Therefore, the $15 M_{\odot}$ estimates can be taken as upper limits, in agreement with calculations of Chen et al. (2006) who find a minimum binary mass of $22 M_{\odot}$ for the protostars within $\mathrm{W} 3\left(\mathrm{H}_{2} \mathrm{O}\right)$. Furthermore, assuming the gas to be in gravito-centrifugal rotation around the two individual cores, our PV plots (see Fig. 10) suggest that the range of 5-15 $M_{\odot}$ is a reasonable estimate for the protostellar masses.

As dust is typically optically thin in the $1.3 \mathrm{~mm}$ wavelength regime and proven to be for this region in particular (Chen et al. 2006), we use the prescription by Hildebrand (1983) to convert the flux density, $F_{\gamma}$, of the continuum observations to a mass. In the form presented by Schuller et al. (2009),

$M=\frac{d^{2} F_{v} R}{B_{v}\left(T_{\mathrm{D}}\right) \kappa_{v}}$

where $R$ is the gas-to-dust mass ratio, $B_{v}\left(T_{\mathrm{D}}\right)$ is the Planck function at a dust temperature of $T_{\mathrm{D}}$, and $\kappa_{v}$ is the dust absorption coefficient. We adopt a gas-to-dust mass ratio of $R=150$ (Draine 2011) and a value of $\kappa_{v}=0.9 \mathrm{~cm}^{2} \mathrm{~g}^{-1}$ for the dust absorption 
coefficient from Ossenkopf \& Henning (1994), corresponding to thin ice mantles after $10^{5} \mathrm{yr}$ of coagulation at a density of $10^{6} \mathrm{~cm}^{-3}$.

High-mass cores, such as the ones we are studying, typically have densities high enough to thermalise the methyl cyanide lines. To check this, using the spontaneous decay rate of $\mathrm{CH}_{3} \mathrm{CN}\left(12_{4}-11_{4}\right)$ obtained from the LAMDA database ${ }^{5}$, $7.65 \times 10^{-4} \mathrm{~s}^{-1}$, and the collision rate of $2.05 \times 10^{-10} \mathrm{~cm}^{3} \mathrm{~s}^{-1}$ with $\mathrm{H}_{2}$ at $140 \mathrm{~K}$ (Green 1986), we calculate the simplified two-level critical density of this line to be $n_{\text {crit }} \approx 3.7 \times 10^{6} \mathrm{~cm}^{-3}$. The effective density, once the radiation field is taken into account, is typically an order of magnitude lower (Evans 1999; Shirley 2015). Following Schuller et al. (2009), the $\mathrm{H}_{2}$ column density is calculated via

$$
N_{\mathrm{H}_{2}}=\frac{S_{v} R}{B_{v}\left(T_{\mathrm{D}}\right) \theta_{\mathrm{B}} \kappa_{v} \mu m_{\mathrm{H}}},
$$

where $S_{v}$ is the peak intensity, $\theta_{\mathrm{B}}$ is the beam solid angle, $\mu$ is the mean molecular weight assumed to be equal to 2.8 , and $m_{\mathrm{H}}$ is the mass of the hydrogen atom. At a temperature of $140 \mathrm{~K}$ and using our continuum intensity of a given position near the centre, we can estimate the $\mathrm{H}_{2}$ column density to be $4.5 \times 10^{24} \mathrm{~cm}^{-2}$. This can be converted to a volume density of $n_{\mathrm{H}_{2}}>7 \times 10^{7} \mathrm{~cm}^{-3}$, assuming the maximum extent of the third dimension to be the plane-of-sky size of the clump ( $4000 \mathrm{AU})$. Therefore, since the density of molecular hydrogen is much higher than the critical density of the lines, the LTE assumption is valid and the rotational temperature map of $\mathrm{CH}_{3} \mathrm{CN}$ obtained from our modelling can be assumed to be tracing the gas kinetic temperature.

Using Eq. (2), our continuum map with its unit converted to Jy pixel $^{-1}$ and the temperature map obtained from XCLASS, assuming dust and gas temperatures are coupled, we obtain a pixel-by-pixel mass density map (see Fig. C.1). Summing over the pixels in our mass density map in the ABD observations, the total mass for $\mathrm{W} 3\left(\mathrm{H}_{2} \mathrm{O}\right)$ is calculated to be $\sim 26.8 M_{\odot}$, with 15.4 $M_{\odot}$ contributed from the core to the east, and $11.4 M_{\odot}$ from the core to the west. Similarly, using the AB observations, we obtain a total mass of $\sim 11.4 M_{\odot}$ for $\mathrm{W} 3\left(\mathrm{H}_{2} \mathrm{O}\right)$, with a core mass of 6.7 and $4.7 M_{\odot}$ from the cores to the east and west, respectively. The effect of spatial filtering of the interferometer is clear in these mass estimates as the exclusion of the D-array data removes more than half of the mass.

Comparing our NOEMA observations to SCUBA-2 $850 \mu \mathrm{m}$ single-dish observations, about $25 \%$ of the flux is filtered out by the interferometer in our $\mathrm{ABD}$ observations (assuming a $v^{-3.5}$ frequency-relation; Beuther et al. 2018), implying that our core mass estimates of 15.4 and $11.4 M_{\odot}$ are lower limits, but nevertheless reasonable. Masses estimated in this manner have contributions from the cores and the disk-like structures and not from any embedded (proto)stars.

\subsection{Toomre stability}

For a differentially rotating disk, the shear force and gas pressure can provide added stability against gravitational collapse. This idea was originally introduced by Safronov (1960) and further quantified by Toomre (1964) for a disk of stars, and has since been used in various applications ranging from planet formation to galaxy dynamics. We investigate the stability of the rotating structures in $\mathrm{W} 3\left(\mathrm{H}_{2} \mathrm{O}\right)$, assuming that they are disks in gravitocentrifugal equilibrium, against axisymmetric instabilities using

5 http://home.strw.leidenuniv.nl/ moldata/ the Toomre $Q$ parameter,

$Q=\frac{c_{\mathrm{S}} \Omega}{\pi G \Sigma}$,

where $c_{\mathrm{S}}$ is the sound speed, and $\Omega$ is the epicyclic frequency of the disk which is equivalent to its angular velocity. The surface density of the disk, $\Sigma$, is calculated by multiplying the column density (Eq. (3)) by the mean molecular weight and mass of the hydrogen atom $\left(\mu m_{\mathrm{H}}\right)$ to convert the number column density to a mass surface density. A thin disk becomes unstable against axisymmetric gravitational instabilities if $Q<1$.

Having obtained a temperature map representative of the kinetic temperature, we are able to calculate the Toomre $Q$ parameter pixel by pixel. In particular, the temperature is used in the calculation of $B_{v}\left(T_{\mathrm{D}}\right)$ and the sound speed,

$c_{\mathrm{s}}=\sqrt{\frac{\gamma k_{\mathrm{B}} T}{\mu m_{\mathrm{H}}}}$,

where $\gamma$ is the adiabatic index with a value of $5 / 3$.

We assume two $10 M_{\odot}$ (proto)stellar objects to be present, one at the position of each of the two continuum peaks (see AB image in Fig. 4). The angular velocity maps are created by adding up mass within concentric circles starting at the position of each core and going outwards. In this way, we incorporate the mass of the rotating structures, calculated via Eq. (2), and the mass of the central objects. The angular velocity of a disk in gravitocentrifugal equilibrium at a radius $r$ is

$\Omega(r)=\sqrt{\frac{G\left(M_{*}+M_{\mathrm{disk}}(r)\right)}{r^{3}}}$,

where the mass of the central object, $M_{*}$, is $10 M_{\odot}$ and $M_{\text {disk }}(r)$ is the gas mass enclosed within $r$. Given the radii involved, this means that in practice, most parts of the maps are dominated by $M_{\text {disk }}$ rather than $M_{*}$.

In Fig. 12, we present our Toomre $Q$ map of W3 $\left(\mathrm{H}_{2} \mathrm{O}\right)$, which is created by stitching together the Toomre $Q$ maps of the two individual cores. The stitching boundary is shown by a solid vertical line and the positions of the two central objects corresponding to our continuum peaks in the $\mathrm{AB}$ image (see Fig. 4) are indicated by stars. While the Toomre $Q$ calculations were performed on the AB-array data, we have drawn the continuum contours from the A-array only observations. The boundary where $Q=1$ is shown by a dashed line.

The most significant factor stabilising the disk against Toomre instability is the high gas temperatures and the fast differential rotation of material closest to the (proto)star; therefore, we find the highest Toomre $Q$ values closest to the presumed locations of the (proto)stars depicted by stars in Fig. 12 as expected. We find low Toomre $Q$ values at the periphery of $\mathrm{W} 3\left(\mathrm{H}_{2} \mathrm{O}\right) \mathrm{E}$ and $\mathrm{W}$, suggesting that both rotating structures are only marginally stable against axisymmetric instabilities. Moreover, W3 $\left(\mathrm{H}_{2} \mathrm{O}\right)$ W shows low Toomre $Q$ values at the positions of additional continuum peaks in the highest resolution A-array data (solid contours in Fig. 12), suggesting further fragmentation at these positions may be possible. As the dimensions of the observed continuum peaks are smaller than the size of our synthesised beam, the validity of this hypothesis needs to be confirmed with higher resolution observations (at $345 \mathrm{GHz}$ ) to be taken with NOEMA.

A Toomre stability analysis performed by Chen et al. (2016) for the high-mass protostar IRAS 20126+4104 showed that the 


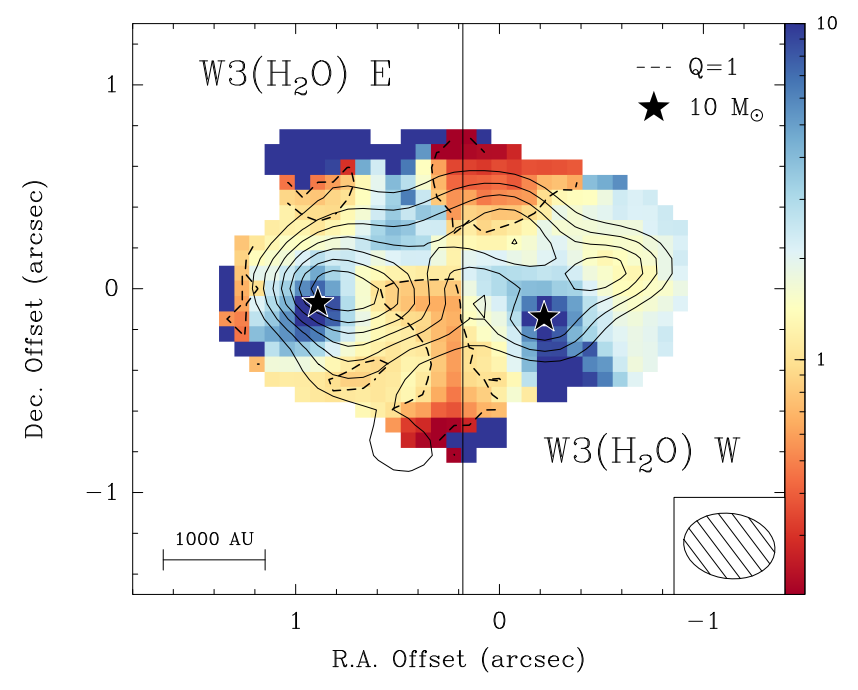

Fig. 12. Toomre $Q$ map obtained by assuming two disk-like structures in gravito-centrifugal rotation about the positions of peak continuum emission as depicted by the two stars, each with a mass of $10 M_{\odot}$. The Toomre $Q$ calculations and the positions of (proto)stars are based on the AB-array data (see Fig. 4) with regions outside the $6 \sigma \mathrm{mm}$ continuum emission masked out. Solid contours correspond to our continuum data in the most extended configuration (A-array), starting at $6 \sigma$ and increasing in steps of $3 \sigma\left(1 \sigma=2.5 \mathrm{mJy}\right.$ beam $\left.^{-1}\right)$. The solid vertical line corresponds to the stitching boundary. The dashed lines correspond to $Q=1$.

massive disk around that protostar is hot and stable to fragmentation, with Toomre $Q>2.8$ at all radii. Hydrodynamic simulations of collapsing protostellar cores by Klassen et al. (2016) find massive accretion disks which evolve to become asymmetrically Toomre-unstable, creating large spiral arms with an increased rate of accretion of material onto the central object. They find that the self-gravity of these spiral arm segments is lower than the tidal forces from the star, causing the spiral arms in their simulations to remain stable against collapse. However, they find that the Toomre conditions combined with the cooling of the disk (Gammie 2001) would potentially yield the formation of a binary companion. Similarly, simulations by Kuiper et al. (2011) see such spiral arms in a massive disk, and simulations by Krumholz et al. (2009) and more recently Meyer et al. (2017, 2018) see disk fragmentation on even smaller spatial scales, on the order of hundreds of AU. Therefore, disk fragmentation scenarios in the high-mass regime are valid from a theoretical stand point.

Among the assumptions that go into this Toomre analysis is the thin-disk approximation. According to Gammie (2001), the critical value of $Q$ for an isothermal disk of finite thickness should be 0.676 (as derived from Goldreich \& Lynden-Bell 1965). Even with such a low critical value, the rotating structures around each core would still show signs of instability in their outskirts. To get an estimate on the importance of disk thickness, following Gammie (2001), the instability condition for a disk with scale height, $H \simeq c_{\mathrm{s}} / \Omega$, can be written as

$$
M_{\text {disk }} \gtrsim \frac{H}{r} M_{*} .
$$

Using our temperature and angular velocity maps, we create a map of the $H / r$ ratio. In this way, we calculate an average value of $H / r \approx 0.3$. Assuming the mass of each (proto)star is roughly $10 M_{\odot}$, we calculate that for the candidate disks to be unstable against gravitational collapse, the mass of the candidate disk must be $M_{\text {disk }} \gtrsim 3 M_{\odot}$. Since the mass estimates for the cores obtained from the continuum maps using Eq. (2) are 15 and $11 M_{\odot}$, the masses are large enough to result in the rotating structures being Toomre-unstable. Therefore, the thickness of the candidate disks does not affect the general interpretation of our results.

The angular velocity maps used in the calculation of the Toomre $Q$ map have been created assuming a face-on geometry for the candidate disks. If the objects are inclined, we would be overestimating the surface density of the candidate disks and the line-of-sight angular velocities. Since these two parameters have the opposite effect on the Toomre $Q$ value (see Eq. (4)), our results are still reasonable. Furthermore, W3 $\left(\mathrm{H}_{2} \mathrm{O}\right)$ E may harbour multiple (proto)stars within it (see Sect. 4.1) such that the possibility of rotational contributions from multi-body rotation would require a different prescription for the epicyclic frequency than what we have assumed.

The estimation of $10 M_{\odot}$ for the central (proto)stars have been deduced from our own and previous mass estimations (see Sect. 4.3), and by examining the emission in the PV plots with Keplerian curves that are just "grazing" the emission. The latter method has the caveat that the observed PV plot is the result of the convolution with the instrumental beam and the line width. Therefore, the real emission is less extended in space and velocity than we observe, and as a result we may be overestimating the mass of the (proto)stars. Performing our Toomre calculations, but with the assumption of $5 M_{\odot}$ (proto)stars, we obtain median Toomre $Q$ values of 1.3 compared to a value of 1.7 in the $10 M_{\odot}$ calculations. Therefore, lower protostellar masses would lead to the decrease in the Toomre $Q$ value, and thus larger unstable regions, making the disk fragmentation scenario even more plausible. On the other hand, as the mass estimates using the bolometric luminosities suggest the central (proto)stellar masses to be in the $15 M_{\odot}$ regime, the median Toomre $Q$ value would be 2.0, with unstable regions still present in the outskirts. The Toomre $Q$ maps created assuming two 5 and $15 M_{\odot}$ (proto) stars at the position of the continuum peaks can be found in Appendix D. The change of $\pm 5 M_{\odot}$ does not strongly affect the Toomre $Q$ maps because we add the mass of the candidate disks, estimated from the continuum emission to be 15 and $11 M_{\odot}$, in rings (see Eq. (6)) and they dominate over an addition or subtraction of $5 M_{\odot}$, especially in the outermost regions. This, together with the root dependence on mass lead to uncertainties in $M_{*}$ to only have a small effect on the Toomre $Q$ results.

We are currently conducting simulated observations from $3 \mathrm{D}$ hydrodynamic simulations to investigate the reliability of our methods in even more depth (Ahmadi et al. in prep.). Our preliminary results on the effect of inclination and (proto)stellar mass on the calculation of Toomre $Q$ maps using hydrodynamic simulations suggest that the Toomre $Q$ value is not strongly dependent on these parameters. A forthcoming paper is dedicated to this topic.

\subsection{Cooling}

For a marginally stable disk $(Q \sim 1)$, the locally unstable regions compress as they start to collapse, providing compressional heating in these regions. This increase in the local thermal pressure can counteract the local collapse and put the disk back into a state of equilibrium such that the region will not fragment unless heat can be dissipated on a short timescale. Through numerical simulations, Gammie (2001) and Johnson \& Gammie (2003) have introduced a cooling parameter, $\beta$, to study the effect of 
cooling for marginally stable thin disks in gravito-centrifugal equilibrium. In their prescription

$\beta=\Omega t_{\text {cooling }}$,

where the cooling time, $t_{\text {cooling }}$, is assumed to be constant and a function of the surface density and internal energy of the disk. If the local collapse needs more than a few orbits, due to a long cooling timescale, a locally collapsing region within the disk can be ripped apart by shear. Therefore, there is a critical $\beta$ value below which a locally collapsing region would be rapidly cooling, and if it is sufficiently self-gravitating, it would be prone to fragmentation. Conversely, values above this critical $\beta$ value would put a marginally stable disk into a self-regulating scenario such that heating acts as a stabilising force and directly counteracts the cooling rate.

The Gammie cooling criterion is relevant only if the disk is marginally stable, with regions that are locally unstable against axisymmetric gravitational instabilities. A Toomre-stable disk will not fragment regardless of how quickly it may be cooling.

This critical value is determined through numerical simulations and varies depending on the heating and cooling recipes and convergence issues. For an overview of these issues, see Kratter \& Lodato (2016) and Baehr et al. (2017). Regardless of the specific numerical simulation issues, it is generally assumed that the critical $\beta$ value ranges between 1 and 5 .

Following the prescription of Klassen et al. (2016),

$t_{\text {cooling }}=\frac{E_{z}}{F_{\text {grey }}}$,

where $E_{z}$ is the column internal energy integrated in the $z$-direction (along the axis of rotation) and $F_{\text {grey }}$ is the radiative flux away from the disk adopting a grey-body approximation. The internal energy of an ideal gas is calculated via

$E_{\text {int }}=c_{v} \rho T$,

where $\rho$ is the volume density and $c_{v}$ is the specific heat capacity defined as

$c_{v}=\frac{N_{\mathrm{A}} k_{\mathrm{B}}}{M_{u}(\gamma-1)}$,

with $N_{\mathrm{A}}$ as Avogadro's number and $M_{u}$ the molar mass. By replacing the volume density with the surface density, the column internal energy is then

$E_{z}=\frac{N_{\mathrm{A}} k_{\mathrm{B}} \Sigma T}{3 M_{u}(\gamma-1)}$.

Knowing the cooling time, which is of the order of months, and the rotation rate at each pixel, we construct a local $\beta$ map. Figure 13 shows the $\beta$ map for the two disks presumed in gravitocentrifugal equilibrium about the positions of peak continuum emission as depicted by stars. The median $\beta$ value is $1.8 \times 10^{-4}$, and the maximum value found in the outskirts is on the order of $10^{-2}$, which is much lower than the critical value of $1-5$. This finding is consistent with that of Klassen et al. (2016) and Meyer et al. (2018) who also find $\beta \ll 1$ in their simulation of collapsing protostellar cores with a mass of $100 M_{\odot}$ at later times. The rotating bodies within $\mathrm{W} 3\left(\mathrm{H}_{2} \mathrm{O}\right)$ are therefore able to cool rapidly and any local collapse induced by the gravitational instabilities in the disks will lead to fragmentation. Rapid cooling in this context means that the cooling timescale is comparable to the local dynamical timescale.

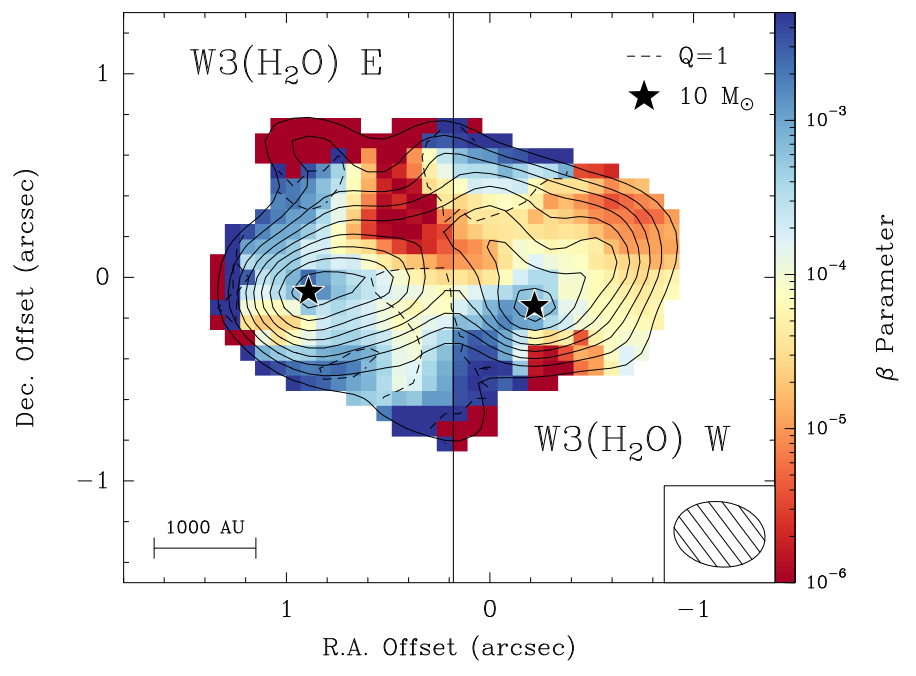

Fig. 13. Map of $\beta$ cooling parameter obtained by assuming two disk-like structures in gravito-centrifugal rotation about the positions of peak continuum emission as depicted by stars. The solid contours correspond to our continuum data in the most extended configuration, starting at $6 \sigma$ and increasing in steps of $3 \sigma\left(1 \sigma=2.5 \mathrm{mJy} \mathrm{beam}^{-1}\right)$. The solid vertical line corresponds to the stitching boundary. The dashed line corresponds to Toomre $Q=1$. Regions outside of the $6 \sigma \mathrm{mm}$ continuum emission contour in the $\mathrm{AB}$ configuration are masked out.

\section{Conclusions and summary}

Our IRAM large programme, CORE, aims to study fragmentation and the kinematics of a sample of 20 high-mass star-forming regions. We have performed a case-study for one of the sources in our sample, the prototypical hot core $\mathrm{W} 3\left(\mathrm{H}_{2} \mathrm{O}\right)$. In this paper we present details of the spectral line setup for our NOEMA observations in the $1.37 \mathrm{~mm}$ band which covers transitions of important tracers of dense gas and disks (e.g. $\mathrm{CH}_{3} \mathrm{CN}, \mathrm{HCOOCH}{ }_{3}, \mathrm{CH}_{3} \mathrm{OH}$ ), inner envelopes (e.g. $\mathrm{H}_{2} \mathrm{CO}$ ), and outflows (e.g. $\left.{ }^{13} \mathrm{CO}, \mathrm{SO}\right)$. We cover the range of $217078.6-220859.5 \mathrm{MHz}$ with a spectral resolution of $2.7 \mathrm{~km} \mathrm{~s}^{-1}$, and eight narrower bands centred on specific transitions to provide higher spectral resolution of $0.5 \mathrm{~km} \mathrm{~s}^{-1}$.

With the aim of studying the fragmentation and kinematic properties of $\mathrm{W} 3\left(\mathrm{H}_{2} \mathrm{O}\right)$, the following is a summary of our findings:

- At an angular resolution of $\sim 0.35$ ( 700 AU at $2 \mathrm{kpc})$, $\mathrm{W} 3\left(\mathrm{H}_{2} \mathrm{O}\right)$ fragments into two cores, which we refer to as W3 $\left(\mathrm{H}_{2} \mathrm{O}\right) \mathrm{E}$ and $\mathrm{W} 3\left(\mathrm{H}_{2} \mathrm{O}\right) \mathrm{W}$, separated by $2300 \mathrm{AU}$, as seen in both line and thermal dust continuum emission.

- Based on the integrated dust continuum emission, W3 $\left(\mathrm{H}_{2} \mathrm{O}\right)$ has a total mass of $\sim 26.8 M_{\odot}$, with $15.4 M_{\odot}$ contributed from $\mathrm{W} 3\left(\mathrm{H}_{2} \mathrm{O}\right) \mathrm{E}$, and $11.4 M_{\odot}$ from W3 $\left(\mathrm{H}_{2} \mathrm{O}\right) \mathrm{W}$.

- On large scales, there is a clear velocity gradient in the east-west direction across $\mathrm{W} 3\left(\mathrm{H}_{2} \mathrm{O}\right)$, spanning $~ 6000 \mathrm{AU}$, attributed to a combination of circumbinary and circumstellar motions. On smaller scales, velocity gradients of a few $\mathrm{km} \mathrm{s}^{-1}$ are observed across each of the cores, perpendicular to the directions of two bipolar molecular outflows, one emanating from each core, as traced by ${ }^{12} \mathrm{CO}$ and ${ }^{13} \mathrm{CO}$. The direction of motion of the gas around the individual cores deviates little from the overall larger scale rotation of $\mathrm{W} 3\left(\mathrm{H}_{2} \mathrm{O}\right)$, suggesting that these motions seen around the cores, which we interpret as rotation, may be inherited from the large-scale rotation. 
- The kinematics of the rotating structure about $\mathrm{W} 3\left(\mathrm{H}_{2} \mathrm{O}\right) \mathrm{W}$ shows differential rotation of material about a (proto)star as deduced from the redshifted part of its PV plot, suggesting that the rotating structure may be a disk-like object. The radio source with a rising spectrum at this position can be attributed to a circumstellar jet or wind ionised by the embedded (proto)star at this position.

- The PV plots of the rotating structure about $\mathrm{W} 3\left(\mathrm{H}_{2} \mathrm{O}\right) \mathrm{E}$ is inconclusive on whether the observed rotation is due to a disk-like rotating object, an unresolved binary (or multiple) system, or a combination of the two.

- We fit the emission of $\mathrm{CH}_{3} \mathrm{CN}\left(12_{K}-11_{K}\right), K=4-6$ and $\mathrm{CH}_{3}{ }^{13} \mathrm{CN}\left(12_{K}-11_{K}\right), K=0-3$ with XCLASS and produce temperature, column density, peak velocity, and velocity dispersion maps. On average, the entire structure is hot $(\sim 165 \mathrm{~K})$ with no particular temperature structure. The column density map of $\mathrm{CH}_{3} \mathrm{CN}$ is double peaked, similar to the continuum and line emission maps, with a median column density of $\sim 1.4 \times 10^{15} \mathrm{~cm}^{-2}$. Close to the centre of the cores the $\mathrm{H}_{2}$ column density is estimated to be $\sim 5 \times 10^{24} \mathrm{~cm}^{-2}$.

- We investigate the axisymmetric stability of the two rotating structures using the Toomre criterion. Our Toomre $Q$ map shows low values in the outskirts of both rotating structures, suggesting that they are unstable to fragmentation. Some regions with low Toomre $Q$ values in the vicinity of $\mathrm{W} 3\left(\mathrm{H}_{2} \mathrm{O}\right) \mathrm{W}$ coincide with unresolved thermal dust continuum peaks (in our highest resolution observations), hinting at the possibility of further fragmentation in this core.

- The Toomre-unstable regions within $\mathrm{W} 3\left(\mathrm{H}_{2} \mathrm{O}\right) \mathrm{E}$ and $\mathrm{W} 3\left(\mathrm{H}_{2} \mathrm{O}\right) \mathrm{W}$ are able to cool rapidly, and any local collapse induced by the gravitational instabilities will lead to further fragmentation.

In this work, we showcase our in-depth analysis for the kinematics and stability of the rotating structures within $\mathrm{W} 3\left(\mathrm{H}_{2} \mathrm{O}\right)$. We showed that high-mass cores can be prone to fragmentation induced by disk instabilities at $\sim 1000$ AU scales, and core fragmentation at larger scales. Therefore, different modes of fragmentation contribute to the final stellar mass distribution of a given region. One question still remains: How universal are these findings? To this end, we aim to benchmark our methods using hydrodynamic simulations and extend our analysis to the full CORE sample in future papers.

Acknowledgements. The authors would like to thank the anonymous referee whose comments helped the clarity of this paper. We also thank Thomas Möller for his technical support with the XCLASS analysis, and Kaitlin Kratter for fruitful discussions regarding the Toomre $Q$ stability analysis. A.A., H.B., J.C.M., and F.B. acknowledge support from the European Research Council under the European Community's Horizon 2020 framework programme (2014-2020) via the ERC Consolidator Grant "From Cloud to Star Formation (CSF)" (project number 648505). R.K. acknowledges financial support via the Emmy Noether Research Group on Accretion Flows and Feedback in Realistic Models of Massive Star Formation funded by the German Research Foundation (DFG) under grant no. KU 2849/3-1. T.Cs. acknowledges support from the Deutsche Forschungsgemeinschaft, DFG via the SPP (priority programme) 1573 "Physics of the ISM". A.P. acknowledges financial support from UNAM and CONACyT, México.

\section{References}

Baehr, H., Klahr, H., \& Kratter, K. M. 2017, ApJ, 848, 40

Behrend, R., \& Maeder, A. 2001, A\&A, 373, 190

Beltrán, M. T., \& de Wit W. J. 2016, A\&ARv, 24, 6

Beltrán, M. T., Cesaroni, R., Neri, R., et al. 2005, A\&A, 435, 901

Beltrán, M. T., Sánchez-Monge, Á., Cesaroni, R., et al. 2014, A\&A, 571, A52

Beuther, H., Schilke, P., Gueth, F., et al. 2002, A\&A, 387, 931

Beuther, H., Zhang, Q., Sridharan, T. K., \& Chen, Y. 2005, ApJ, 628, 800
Beuther, H., Zhang, Q., Hunter, T. R., Sridharan, T. K., \& Bergin, E. A. 2007, A\&A, 473, 493

Beuther, H., Mottram, J. C., Ahmadi, A., et al. 2018, A\&A, 617, A100

Boehm-Vitense, E. 1981, ARA\&A, 19, 295

Bontemps, S., Motte, F., Csengeri, T., \& Schneider, N. 2010, A\&A, 524, A18

Carrasco-González, C., Galván-Madrid, R., Anglada, G., et al. 2012, ApJ, 752, L29

Cesaroni, R., Galli, D., Lodato, G., Walmsley, C. M., \& Zhang, Q. 2007, in Protostars and Planets V, eds. B. Reipurth, D. Jewitt, \& K. Keil (Tucson, AZ: Arizona University Press), 197

Cesaroni, R., Sánchez-Monge, Á., Beltrán, M. T., et al. 2017, A\&A, 602, A59

Chen, H.-R., Welch, W. J., Wilner, D. J., \& Sutton, E. C. 2006, ApJ, 639, 975

Chen, H.-R. V., Keto, E., Zhang, Q., et al. 2016, ApJ, 823, 125

Clark, B. G. 1980, A\&A, 89, 377

Davies, B., Hoare, M. G., Lumsden, S. L., et al. 2011, MNRAS, 416, 972

Draine, B. T. 2011, Physics of the Interstellar and Intergalactic Medium (Princeton: Princeton University Press)

Dreher, J. W., \& Welch, W. J. 1981, ApJ, 245, 857

Evans, II, N. J. 1999, ARA\&A, 37, 311

Fallscheer, C., Beuther, H., Zhang, Q., Keto, E., \& Sridharan, T. K. 2009, A\&A, 504,127

Feng, S., Beuther, H., Henning, T., et al. 2015, A\&A, 581, A71

Frank, A., Ray, T. P., Cabrit, S., et al. 2014, in Protostars and Planets VI, eds. H. Beuther, R. Klessen, C. Dullemond, \& T. Henning (Tucson, AZ: University of Arizona Press), 451

Gammie, C. F. 2001, ApJ, 553, 174

Goldreich, P., \& Lynden-Bell, D. 1965, MNRAS, 130, 125

Green, S. 1986, ApJ, 309, 331

Hachisuka, K., Brunthaler, A., Menten, K. M., et al. 2006, ApJ, 645, 337

Henkel, C., Wilson, T. L., \& Bieging, J. 1982, A\&A, 109, 344

Hildebrand, R. H. 1983, QJRAS, 24, 267

Ilee, J. D., Cyganowski, C. J., Nazari, P., et al. 2016, MNRAS, 462, 4386

Johnson, B. M., \& Gammie, C. F. 2003, ApJ, 597, 131

Johnston, K. G., Robitaille, T. P., Beuther, H., et al. 2015, ApJ, 813, L19

Kahn, F. D. 1974, A\&A, 37, 149

Keto, E., \& Wood, K. 2006, ApJ, 637, 850

Keto, E. R., Welch, W. J., Reid, M. J., \& Ho, P. T. P. 1995, ApJ, 444, 765

Klassen, M., Pudritz, R. E., Kuiper, R., Peters, T., \& Banerjee, R. 2016, ApJ, 823,

Kratter, K., \& Lodato, G. 2016, ARA\&A, 54, 271

Krumholz, M. R., Klein, R. I., McKee, C. F., Offner, S. S. R., \& Cunningham, A. J. 2009, Science, 323, 754

Kuiper, R., \& Yorke, H. W. 2013, ApJ, 772, 61

Kuiper, R., Klahr, H., Beuther, H., \& Henning, T. 2010, ApJ, 722, 1556

Kuiper, R., Klahr, H., Beuther, H., \& Henning, T. 2011, ApJ, 732, 20

Kurtz, S., Churchwell, E., \& Wood, D. O. S. 1994, ApJS, 91, 659

Leurini, S., Codella, C., Zapata, L., et al. 2011, A\&A, 530, A12

Loren, R. B., \& Mundy, L. G. 1984, ApJ, 286, 232

Martins, F., Schaerer, D., \& Hillier, D. J. 2005, A\&A, 436, 1049

Matsumoto, T., \& Hanawa, T. 2003, ApJ, 595, 913

Maud, L. T., Moore, T. J. T., Lumsden, S. L., et al. 2015, MNRAS, 453, 645

Meyer, D. M.-A., Vorobyov, E. I., Kuiper, R., \& Kley, W. 2017, MNRAS, 464, L90

Meyer, D. M.-A., Kuiper, R., Kley, W., Johnston, K. G., \& Vorobyov, E. 2018, MNRAS, 473, 3615

Möller, T., Endres, C., \& Schilke, P. 2017, A\&A, 598, A7

Motte, F., Bontemps, S., \& Louvet, F. 2018, ARA\&A, 56, 41

Mottram, J. C., Hoare, M. G., Urquhart, J. S., et al. 2011, A\&A, 525, A149

Mottram, J. C., et al. 2018, A\&A, submitted

Müller, H. S. P., Thorwirth, S., Roth, D. A., \& Winnewisser, G. 2001, A\&A, 370 L49

Müller, H. S. P., Schlöder, F., Stutzki, J., \& Winnewisser, G. 2005, J. Mol. Struct., 742,215

Norberg, P., \& Maeder, A. 2000, A\&A, 359, 1025

Ohashi, N., Hayashi, M., Ho, P. T. P., \& Momose, M. 1997, ApJ, 475, 211

Ossenkopf, V., \& Henning, T. 1994, A\&A, 291, 943

Palau, A., Fuente, A., Girart, J. M., et al. 2013, ApJ, 762, 120

Palau, A., Ballesteros-Paredes, J., Vázquez-Semadeni, E., et al. 2015, MNRAS, 453, 3785

Peters, T., Banerjee, R., Klessen, R. S., et al. 2010, ApJ, 711, 1017

Pickett, H. M., Poynter, R. L., Cohen, E. A., et al. 1998, J. Quant. Spectr. Rad. Transf., 60, 883

Pudritz, R. E., Ouyed, R., Fendt, C., \& Brandenburg, A. 2007, in Protostars and Planets V, eds. B. Reipurth, D. Jewitt, \& K. Keil (Tucson, AZ: University of Arizona Press), 277

Qin, S.-L., Schilke, P., Wu, J., et al. 2015, ApJ, 803, 39 
Reid, M. J., Argon, A. L., Masson, C. R., Menten, K. M., \& Moran, J. M. 1995, ApJ, 443, 238

Safronov, V. S. 1960, Ann. Astrophys., 23, 979

Sánchez-Monge, Á. 2011, PhD Thesis, Universitat de Barcelona, Spain

Sánchez-Monge, Á., Cesaroni, R., Beltrán, M. T., et al. 2013, A\&A, 552, L10

Schuller, F., Menten, K. M., Contreras, Y., et al. 2009, A\&A, 504, 415

Shchekinov, Y. A., \& Sobolev, A. M. 2004, A\&A, 418, 1045

Shirley, Y. L. 2015, PASP, 127, 299

Steer, D. G., Dewdney, P. E., \& Ito, M. R. 1984, A\&A, 137, 159

Tobin, J. J., Hartmann, L., Bergin, E., et al. 2012, ApJ, 748, 16

Toomre, A. 1964, ApJ, 139, 1217

Turner, J. L., \& Welch, W. J. 1984, ApJ, 287, L81

Williams, J. P., Blitz, L., \& McKee, C. F. 2000, in Protostars and Planets IV, eds. V. Mannings, A. Boss, \& S. Russell (Tucson, AZ: Arizona University Press), 97

Wilner, D. J., Reid, M. J., \& Menten, K. M. 1999, ApJ, 513, 775

Wolfire, M. G., \& Cassinelli, J. P. 1987, ApJ, 319, 850

Wyrowski, F., Schilke, P., Walmsley, C. M., \& Menten, K. M. 1999, ApJ, 514, L43

Xu, Y., Reid, M. J., Zheng, X. W., \& Menten, K. M. 2006, Science, 311, 54

Yorke, H. W., \& Sonnhalter, C. 2002, ApJ, 569, 846

Zapata, L. A., Rodríguez-Garza, C., Rodríguez, L. F., Girart, J. M., \& Chen, H.-R. 2011, ApJ, 740, L19

Zhang, Q., Ho, P. T. P., \& Ohashi, N. 1998, ApJ, 494, 636

${ }^{1}$ Max Planck Institute for Astronomy, Königstuhl 17, 69117 Heidelberg, Germany e-mail: ahmadi@mpia.de

2 International Max Planck Research School for Astronomy and Cosmic Physics at the University of Heidelberg (IMPRS-HD)

3 IRAM, 300 Rue de la Piscine, Domaine Universitaire de Grenoble, 38406 St.-Martin-d'Hères, France

${ }^{4}$ Institute of Astronomy and Astrophysics, University of Tübingen, Auf der Morgenstelle 10, 72076 Tübingen, Germany

5 Department of Physics and Astronomy, McMaster University, 1280 Main St. W, Hamilton, ON L8S 4M1, Canada
${ }^{6}$ I. Physikalisches Institut, Universität zu Köln, Zülpicher Str. 77, 50937 Köln, Germany

7 Harvard-Smithsonian Center for Astrophysics, 160 Garden St, Cambridge, MA 02420, USA

${ }^{8}$ INAF, Osservatorio Astrofisico di Arcetri, Largo E. Fermi 5, 50125 Firenze, Italy

9 Laboratoire d'Astrophysique de Bordeaux - UMR 5804, CNRS Université Bordeaux 1, BP 89, 33270 Floirac, France

${ }^{10}$ Max-Planck-Institut für Radioastronomie, Auf dem Hügel 69, 53121 Bonn, Germany

${ }^{11}$ Max-Planck-Institut für Extraterrestrische Physik, Gissenbachstrasse 1, 85748 Garching, Germany

12 Instituto de Radioastronomía y Astrofísica, Universidad Nacional Autonóma de México, PO Box 3-72, 58090 Morelia, Michoacan, Mexico

13 School of Physics \& Astronomy, E.C. Stoner Building, The University of Leeds, Leeds LS2 9JT, UK

${ }^{14}$ UK Astronomy Technology Centre, Royal Observatory Edinburgh, Blackford Hill, Edinburgh EH9 3HJ, UK

15 INAF - Osservatorio Astronomico di Cagliari, Via della Scienza 5, Selargius CA 09047, Italy

16 Astrophysics Research Institute, Liverpool John Moores University, 146 Brownlow Hill, Liverpool L3 5RF, UK

${ }^{17}$ Leiden Observatory, Leiden University, PO Box 9513, 2300 RA Leiden, The Netherlands

18 CNRS, Institut de Planétologie et d'Astrophysique de Grenoble, Université Grenoble Alpes, 38000 Grenoble, France

19 Max-Planck-Institut für Astrophysik, Karl-Schwarzschild-Str. 1, 85748 Garching, Germany

${ }^{20}$ School of Physics and Astronomy, Cardiff University, Cardiff CF24 3AA, UK

${ }^{21}$ Centre for Astrophysics and Planetary Science, University of Kent, Canterbury CT2 7NH, UK

${ }^{22}$ SOFIA Science Center, Deutsches SOFIA Institut, NASA Ames Research Center, Moffett Field, CA 94035, USA

${ }^{23}$ Universidad Autónoma de Chile, Av. Pedro de Valdivia 425, Santiago, Chile 


\section{Appendix A: Moment maps}

In this section, we present zeroth (Fig. A.1), first (Fig. A.2), and second (Fig. A.3) moment maps of various detected lines in the narrow-band receiver for $\mathrm{W} 3\left(\mathrm{H}_{2} \mathrm{O}\right)$ (top panels) and $\mathrm{W} 3(\mathrm{OH})$ (bottom panels) in the combined A-, B-, and D-array observations. All moment maps have been created inside regions where the $\mathrm{S} / \mathrm{N}$ is greater than $5 \sigma$.
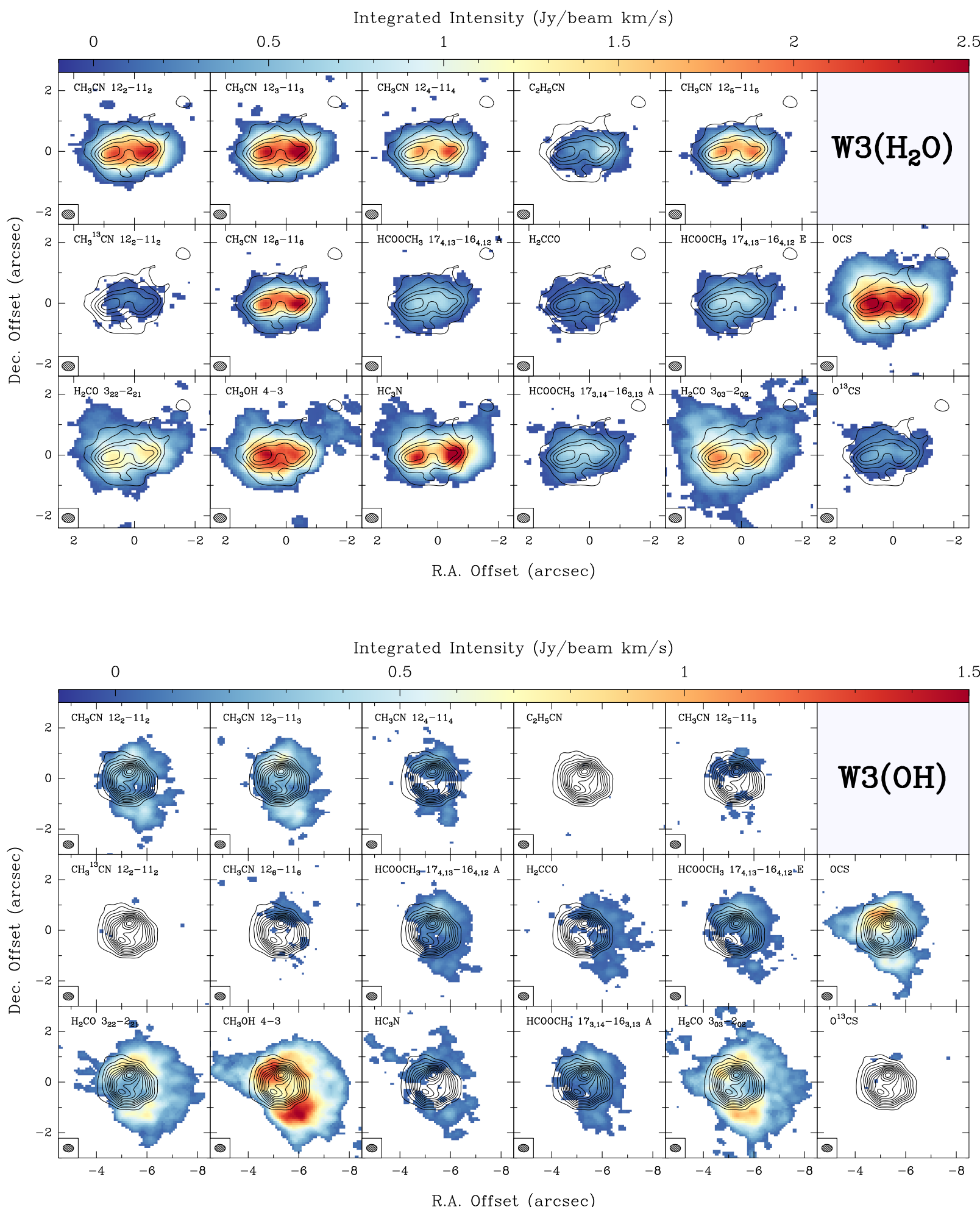

Fig. A.1. Integrated intensity (zeroth moment) maps of most important lines covered in the narrow-band receiver for the observations in the ABD configuration for $\mathrm{W} 3\left(\mathrm{H}_{2} \mathrm{O}\right)$ (top panel) and $\mathrm{W} 3(\mathrm{OH})$ (bottom panel). The solid contours correspond to the dust continuum and start at and increase

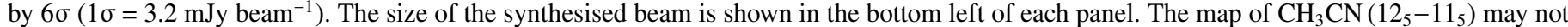
be accurate because it is blended with other lines. 

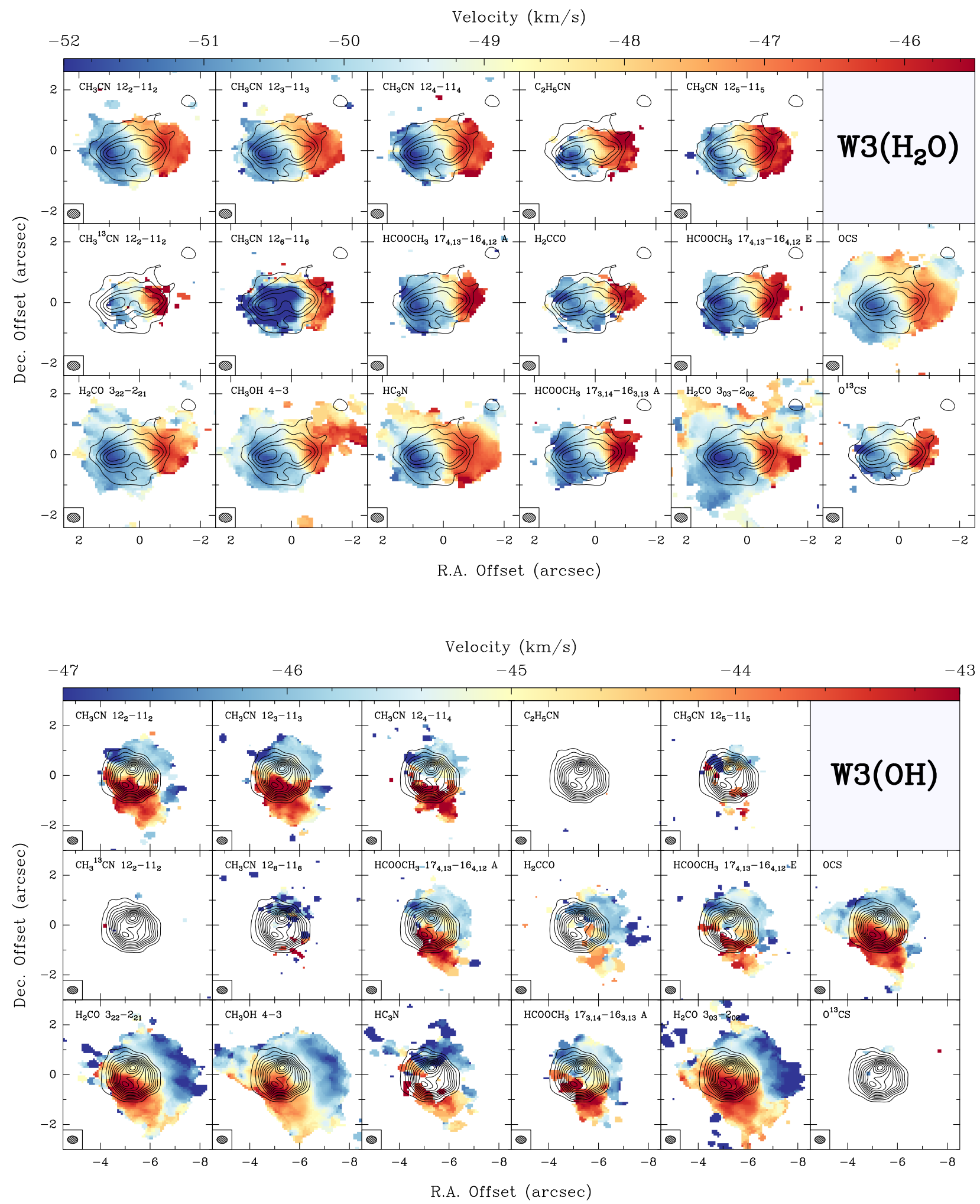

Fig. A.2. Intensity-weighted peak velocity (first moment) maps of most important lines covered in the narrow-band receiver for the observations in the $\mathrm{ABD}$ configuration for $\mathrm{W} 3\left(\mathrm{H}_{2} \mathrm{O}\right)$ (top panel) and $\mathrm{W} 3(\mathrm{OH})$ (bottom panel). The solid contours correspond to the dust continuum and start at and increase by $6 \sigma\left(1 \sigma=3.2 \mathrm{mJy}_{\text {beam }}{ }^{-1}\right)$. The size of the synthesised beam is shown in the bottom left of each panel. The map of $\mathrm{CH}_{3} \mathrm{CN}\left(12_{5}-11_{5}\right)$ may not be accurate because it is blended with other lines. 

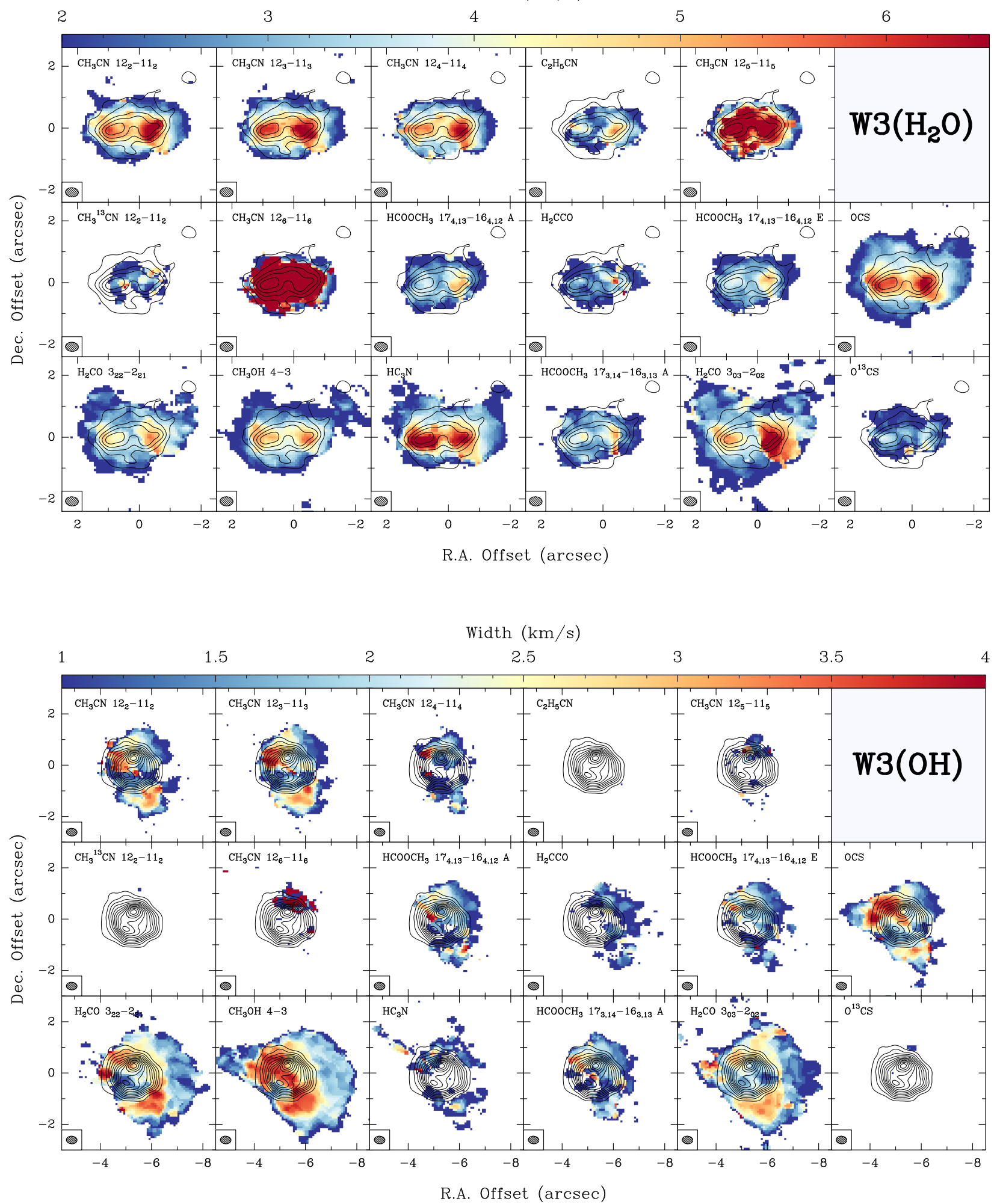

Fig. A.3. Root mean square velocity dispersion (second moment) maps of most important lines covered in the narrow-band receiver for the observations in the ABD configuration for $\mathrm{W} 3\left(\mathrm{H}_{2} \mathrm{O}\right)$ (top panel) and $\mathrm{W} 3(\mathrm{OH})$ (bottom panel). The solid contours correspond to the dust continuum and start at and increase by $6 \sigma\left(1 \sigma=3.2 \mathrm{mJy}_{\text {beam }}^{-1}\right)$. The size of the synthesised beam is shown in the bottom left of each panel. The map of $\mathrm{CH}_{3} \mathrm{CN}\left(12_{5}-11_{5}\right)$ may not be accurate because it is blended with other lines. 


\section{Appendix B: Details of XCLASS fitting}

XCLASS explores the parameter space (source size, column density, temperature, linewidth, and peak velocity) using as many algorithms as the user demands, and stops when the maximum number of iterations has been reached. In our analysis, we employed a combination of the Genetic and Levenberg-Marquardt algorithms ${ }^{6}$, and an isothermal model such that one temperature is used to reproduce the observed population of lines for a given species in a given spectrum. We assumed that the size of the source was larger than the beam and therefore did not fit the beam filling factor parameter. Our initial fits to the spectra of $\mathrm{CH}_{3} \mathrm{CN}$ included the full $K=0-6$ ladder, along with its $\mathrm{CH}_{3}{ }^{13} \mathrm{CN}\left(12_{K}-11_{K}\right), K=0-3$ isotopologue, prescribing the ${ }^{12} \mathrm{C} /{ }^{13} \mathrm{C}$ ratio to be 76 , which is consistent with the findings of Henkel et al. (1982) and the calculations of Qin et al. (2015) for $\mathrm{W} 3\left(\mathrm{H}_{2} \mathrm{O}\right)$. The top panel of Fig. B.1 shows an example of the best-fit spectrum for one pixel in the AB observations, which yielded a high rotational temperature of $835 \mathrm{~K}$. The lower intensities of the low- $K$ lines of $\mathrm{CH}_{3} \mathrm{CN}$ compared to the transitions higher on the $K$-ladder indicates that the low- $K$ transitions are optically thick. Furthermore, the fits to the optically thinner high- $K$ lines are not satisfactory. Repeating the same procedure, but only fitting the $\mathrm{CH}_{3} \mathrm{CN}\left(12_{K}-11_{K}\right), K=4-6$ lines along with the $\mathrm{CH}_{3}{ }^{13} \mathrm{CN}\left(12_{K}-11_{K}\right), K=0-3$ isotopologues results in a better fit to these lines for a lower rotational temperature of $207 \mathrm{~K}$ (see bottom panel of Fig. B.1). These findings are in agreement with line fitting analysis of Feng et al. (2015) in Orion $\mathrm{KL}$ in which they find that fitting all $\mathrm{CH}_{3} \mathrm{CN}$ lines, assuming they are optically thin and in LTE, yields higher temperatures than when fitting them with an optical depth correction (see their Fig. 8). The exclusion of the optically thick and low-energy lower- $K$ lines along with the inclusion of the ${ }^{13} \mathrm{C}$ isotopologues, although barely detected, allows the software to avoid prioritising the fitting of these optically thick lines and therefore derives the temperature more accurately. This finding is also related to the existence of temperature and density gradients which our one-component model cannot properly reproduce. As lower- $K$ lines are more easily excited than the higher- $K$ lines, they can probe the temperature in the envelope and in the disk surface, while the higher- $K$ lines are better at tracing the disk and may not be as excited in the envelope. In fact, the reason why the brightness temperature of the low- $K$ lines is lower than the high- $K$ lines may be due to self-absorption of the photons from the warmer inner region by molecules in the cooler envelope material between the disk and the observer. Another explanation can be that the optically thick low- $K$ transitions are more extended and therefore may be partially resolved out.

\section{Appendix C: Mass density map}

In this section we present the mass density map for $\mathrm{W} 3\left(\mathrm{H}_{2} \mathrm{O}\right)$ in Fig. C.1, which was constructed using Eq. (2) with the continuum map converted to Jy pixel $^{-1}$ units, and the temperature map obtained from modelling $\mathrm{CH}_{3} \mathrm{CN}\left(12_{K}-11_{K}\right), K=4-6$ lines with XCLASS. This map is used in the calculation of the angular velocity in the construction of the Toomre $Q$ map.

In Fig. D.1, we present Toomre $Q$ maps created assuming either two $5 M_{\odot}$ (proto)stars at the positions of the two continuum peaks (top panel), or two $15 M_{\odot}$ (proto)stars (bottom panel).

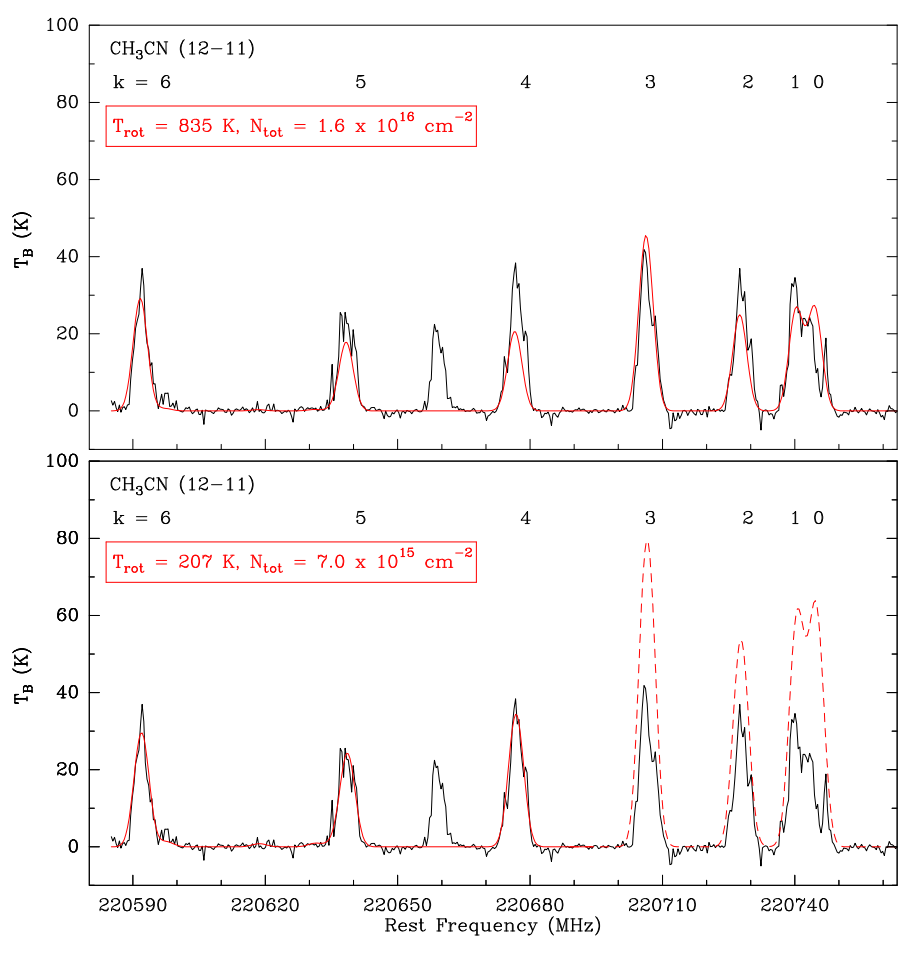

Fig. B.1. Top panel: spectrum of a given pixel (in black) along with the XCLASS fit for $\mathrm{CH}_{3} \mathrm{CN}\left(12_{K}-11_{K}\right), K=0-6$ and $\mathrm{CH}_{3}{ }^{13} \mathrm{CN}\left(12_{K}-11_{K}\right), K=0-3$ lines (in solid red). The corresponding fit parameters are provided in the panel. Bottom panel: spectrum of the same pixel as above (in black) with the XCLASS fit for $\mathrm{CH}_{3} \mathrm{CN}\left(12_{K}-11_{K}\right), K=4-6$ and $\mathrm{CH}_{3}{ }^{13} \mathrm{CN}\left(12_{K}-11_{K}\right), K=0-3$ (in solid red). The dashed red line corresponds to the predicted spectrum for the $\mathrm{CH}_{3} \mathrm{CN}$ lines that were not used in the fitting process. This shows that the exclusion of the low- $K$ lines in the fitting process allows XCLASS to provide a better fit for the optically thinner high- $K$ lines. The bright line detected between the $K=4$ and 5 components is $\mathrm{C}_{2} \mathrm{H}_{5} \mathrm{CN}$.

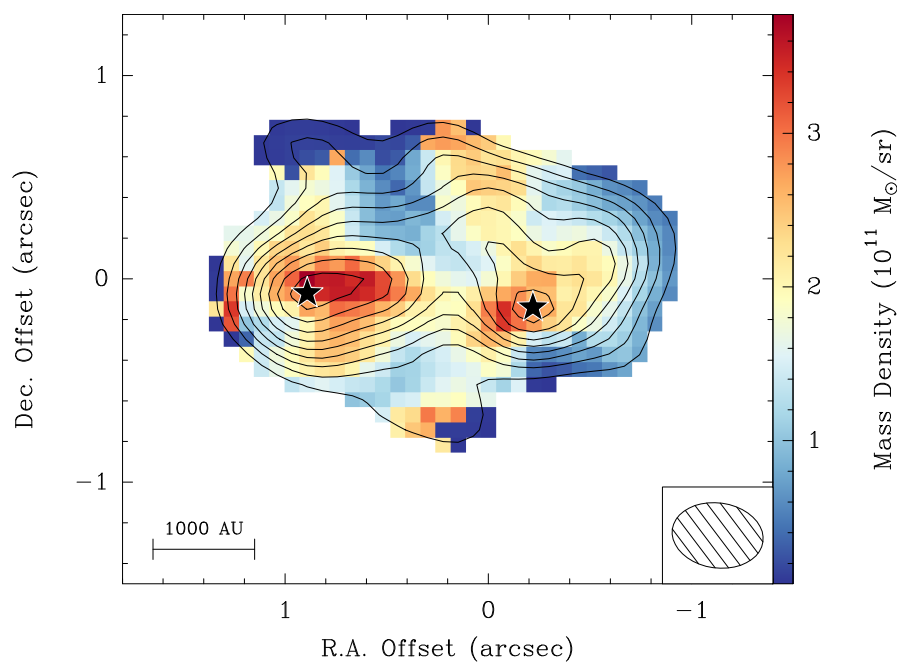

Fig. C.1. Mass density map obtained using the temperature and continuum maps in Eq. (2). The solid contours correspond to our continuum observations in the $\mathrm{AB}$ configuration, starting at $6 \sigma$ and increasing in steps of $3 \sigma\left(1 \sigma=2.5 \mathrm{mJy}^{\text {beam }}{ }^{-1}\right)$. Each of the peak continuum positions, as depicted by stars, is expected to host at least one $10 M_{\odot}$ (proto)star or more lower mass sources.

\footnotetext{
6 See the XCLASS manual for algorithm descriptions.
} 
Appendix D: Toomre $Q$ maps
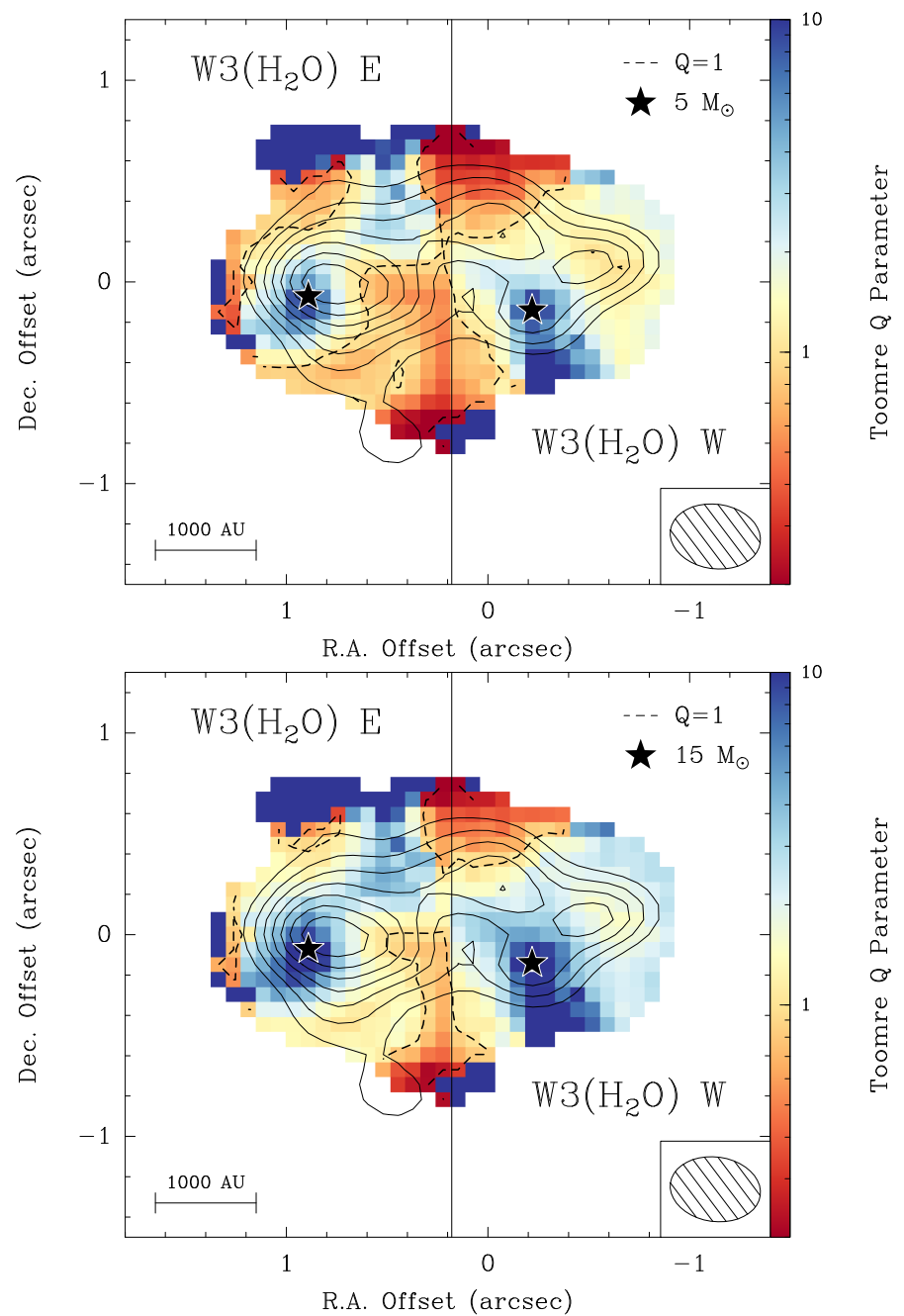

Fig. D.1. Toomre $Q$ map obtained by assuming two disks in gravitocentrifugal rotation about two $5 M_{\odot}$ (proto)stars (top panel) and two $15 M_{\odot}$ (proto)stars (bottom panel) at the positions of peak continuum emission as depicted by the two stars. The solid contours correspond to our continuum data in the most extended configuration, starting at $6 \sigma$ and increasing in steps of $3 \sigma\left(1 \sigma=2.5 \mathrm{mJy}\right.$ beam $\left.^{-1}\right)$. The solid vertical line corresponds to the stitching boundary. The dashed line corresponds to $Q=1$. Regions outside of the $6 \sigma \mathrm{mm}$ continuum emission contour in the $\mathrm{AB}$ configuration are masked out. 